\title{
The long-term impact of ranking algorithms in growing networks
}

\author{
Shilun Zhanga ${ }^{\mathrm{a}}$, Matúš Medo ${ }^{\mathrm{a}, \mathrm{b}, \mathrm{c}}$, Linyuan Lü ${ }^{\mathrm{a}, \mathrm{d}}$, Manuel Sebastian Mariani ${ }^{\mathrm{a}, \mathrm{e}}$ \\ ${ }^{a}$ Institute of Fundamental and Frontier Sciences, University of Electronic Science and \\ Technology of China, 610051 Chengdu, PR China \\ ${ }^{b}$ Department of Radiation Oncology, Inselspital, Bern University Hospital and University of \\ Bern, 3010 Bern, Switzerland \\ ${ }^{c}$ Department of Physics, University of Fribourg, 1700 Fribourg, Switzerland \\ ${ }^{d}$ Alibaba Research Center for Complexity Sciences, Hangzhou Normal University, 311121 \\ Hangzhou, PR China \\ ${ }^{e}$ URPP Social Networks, Universität Zürich, 8050 Zürich, Switzerland
}

\begin{abstract}
When we search online for content, we are constantly exposed to rankings. For example, web search results are presented as a ranking, and online bookstores often show us lists of best-selling books. While popularity-based ranking algorithms (like Google's PageRank) have been extensively studied in previous works, we still lack a clear understanding of their potential systemic consequences. In this work, we fill this gap by introducing a new model of network growth that allows us to compare the properties of the networks generated under the influence of different ranking algorithms. We show that by correcting for the omnipresent age bias of popularity-based ranking algorithms, the resulting networks exhibit a significantly larger agreement between the nodes' inherent quality and their long-term popularity, and a less concentrated popularity distribution. To further promote popularity diversity, we introduce and validate a perturbation of the original rankings where a small number of randomly-selected nodes are promoted to the top of the ranking. Our findings move the first steps toward a model-based understanding of the long-term impact of popularity-based ranking algorithms, and could be used as an informative tool for the design of improved information filtering tools.
\end{abstract}

Keywords: Complex networks, Ranking, Popularity and quality, Popularity inequality, Algorithmic bias

Email addresses: linyuan.1v@uestc.edu.cn (Linyuan Lü), manuel.mariani@business.uzh.ch (Manuel Sebastian Mariani) 


\section{Introduction}

Ranking algorithms allow us to efficiently sort the massive amount of online information, aiming to quickly provide us with the relevant items that fit our needs. Due to the ubiquity of ranking, the implications of ranking-based information filtering tools such as search engines [4] and recommendation systems [23] for our society are widely debated $[3,6,9,17]$. Even when free from apparent manipulation, the rankings that we are exposed to in online platforms and information repositories are influenced by social processes and, at the same time, influence social processes themselves [45]. To provide a few examples, rankings can heavily impact on the eventual popularity of movies and songs in cultural markets [43], affect the attention received by products in online e-commerce platforms [18, 48, 59], increase the sales of top-ranked dishes in restaurants [5], and even influence the choices of undecided electors and, as a result, the outcome of political elections [11]. Understanding the potential systemic impact of ranking algorithms and correcting their potential flaws becomes therefore a critical issue in diverse contexts.

A robust finding in previous experiments of diverse nature [5, 6, 13, 21, 42, 43] is that the current ranking position of an item (or, generally, its current popularity) heavily influences its eventual popularity or success. As a consequence, one of the key challenges is to assess whether in a given system, the final popularity of an item is a reliable proxy for its "quality" or "fitness", where quality is interpreted as the success the item would have in absence of social influence mechanisms [43]. In other words, it becomes critical to determine whether the adoption of a given ranking algorithm by a given system allows high-quality nodes to experience larger success than low-quality nodes. If this is not the case, we may conclude that the adopted ranking algorithm has a negative impact on the system, as it may prevent high-quality nodes from becoming popular and allow low-quality nodes to stand out.

Cho [6] pointed out that ranking algorithms and search engines that favor already popular items create a strong "popularity bias" [13] - also dubbed as "searchengine bias" [6], and "googlearchy" [17] - such that only the already popular nodes can receive substantial attention in the next future, whereas recent high-quality nodes go essentially unnoticed [6]. This bias can amplify initial differences between the items' popularity, leading some items to a disproportionately high popularity regardless of their quality. O'Madadhain et al. [35] emphasized that static ranking algorithms (like Google's PageRank [4]) are based on time-aggregate network representations and, for this reason, they do not respect the sequence of events that led to the formation of the network. To solve this issue, they introduced [35] and validated [36] a network-based ranking algorithm, called EventRank, that takes into account the detailed sequence of events, in a similar spirit as the recent literature on temporal networks [22, 46, 47, 57]. Salganik et al. [42, 43] found that in artificial cultural markets, showing the items' ranking by popularity to con- 
sumers significantly impacts on the items' final popularity. Recent studies [37, 56] emphasized the individuals' limited attention as a determinant for the viral popularity of low-quality items. Other recent works focused on modelling the interplay between quality/talent and popularity for diverse types of agents, including websites [20], scientific papers [30, 55], researchers [29, 50], and bestseller books [58]. Both model-based [20,30] and experimental results [43] indicate that in presence of social influence, the relation between popularity and quality is highly non-linear, meaning that a small variation of quality leads to large variations in popularity.

While both the relation between popularity and quality and the search-engine bias in the Web have attracted considerable attention from previous research, the abilities of different ranking algorithms to promote quality in a given system are typically not compared with each other. The main reason is that the intrinsic node quality is typically inaccessible in the real world, and we lack a clear understanding of how the interplay between ranking and quality shapes the growth of a given system. As a result, we still lack a general framework to assess the long-term impact of different ranking algorithms.

The main goal of this paper is to move the first steps toward filling this gap. To this end, building on existing models of network growth [12] and popularity dynamics [7], we introduce a growing directed-network model where each node, when choosing the nodes to point to, is driven either by the results of a given adopted ranking algorithm or by quality. The nodes' sensitivity to quality is a homogeneous parameter of the model [7]. Crucially, different adopted ranking algorithms lead to different properties of the final network. We use the model to address the following questions: Will a given algorithm facilitate or impede the success of high-quality nodes in the system? Is a given algorithm useful in discovering high-quality nodes? Does it lead to uneven, highly-concentrated popularity distribution?

We postulate that a good ranking algorithm should lead to a network where: (1) node long-term popularity strongly correlates with their quality (quality promotion); (2) nodes' score strongly correlates with their quality (quality detection); (3) the node popularity distribution is relatively diverse (diversity promotion). The quality promotion and detection properties favor the algorithms that help the system to improve the popularity-quality correlation (quality promotion) [7] and help the nodes to find high-quality nodes (quality detection) [26]. The diversity promotion favors the algorithms that distribute popularity more evenly across the nodes, making it easier for the nodes to find quality nodes that are not among the most popular ones [61].

In fact, the synthetic networks generated with our ranking-based growth model can be interpreted as benchmark graphs for ranking algorithms. Our focus on quality promotion, quality detection, and diversity promotion makes our validation framework for ranking algorithm fundamentally different from existing benchmarking techniques which focus on the ability of the algorithms to identify structurally 
vital nodes [31], find those nodes that maximize the reach of a spreading process [24, 38], single out expert-selected important nodes [27, 28], or respect sets of axioms $[1,2,36,44]$.

We find that in networks that adopt cumulative popularity (as measured by the number of incoming links - node indegree [32]) as the ranking algorithm, the correlation between node popularity and quality strongly depends not only on the nodes' sensitivity to quality, but also on their willingness to select low-ranked nodes ("exploration cost" in [7]). A popularity metric that is not biased by node age [27, 33] (called rescaled indegree in [27]) leads to networks where both the final nodes' popularity and the node score are significantly better correlated with node quality, and the final popularity distribution is significantly more diverse. Interestingly, when the exploration cost is large, networks that adopted a random ranking of the nodes exhibit even higher indegree-quality correlation than networks that adopted a popularity-based ranking: while popularity-based ranking algorithms are always useful for the nodes to discover high-quality content, they may accelerate the dissemination of low-quality content when individuals rely too heavily on them.

To further promote popularity diversity, we introduce and validate a ranking algorithm - the ranking by rescaled indegree with random promotion - where the original ranking by rescaled indegree is "perturbed" by promoting a small number of randomly-selected nodes to the top-10 or the top-20 of the ranking. Such a perturbation has a deterministic component (the number of nodes that are promoted is fixed) and a noisy one (the promoted nodes are chosen at random), and it allows us to study the impact of a small amount of noise on systemic properties, in a similar spirit as previous studies that investigated the impact of noise on democratic consensus promotion in animal groups [8], dynamical influence detection [19], and the performance of human groups in coordination problems [49]. We find that with respect to the ranking by rescaled indegree, the ranking by rescaled indegree with random promotion generates networks with increased popularity diversity. Intriguingly, we find that the random promotion can have a marginal or a negative impact on the agreement between nodes' final popularity and quality, depending on the model parameters.

This work provides the first systematic comparison of network-based ranking algorithms with respect to their long-term systemic effects. It complements the question of whether a given ranking algorithm is able to detect important nodes $[22,24]$ with the question of whether high-quality nodes will stand out in a system that adopted that given metric. It reveals that suppressing the bias by node age of popularity-based metrics is beneficial to quality promotion, detection, and popularity diversity.

The manuscript is organized as follows. Section 2 introduces our model of network growth together with the ranking algorithms considered here, and the 
ranking evaluation criteria. Section 3 presents the results of our numerical simulations both for the basic model (Sections 3.1-3.4) and for a variant of the model with node removal (Section 3.5), together with an application of our model to a real information network of scientific papers (Section 3.6). Section 4 is dedicated to a discussion of our results and their implications for algorithmic evaluation and the quality-popularity relation in information systems. Appendices A-B conclude the main text. The Supplementary Material (SM) file is available online - figures whose label contains an "S" (e.g., Fig. S1) can be found in the SM file.

\section{Model and ranking algorithms}

In this Section, we introduce the model of network growth (Section 2.1), the ranking algorithms considered in this paper (Section 2.2), and the metrics used to evaluate the long-term impact of ranking algorithms (Section 2.3).

\subsection{The model of network growth}

We focus here on monopartite directed networks. Our model is meant to represent a social or information network where the number of nodes grows with time. In the model, each node $i$ is endowed with a quality parameter $q_{i}$ which quantifies its attractiveness to new incoming connections in absence of ranking influence. Before generating each network, we choose the ranking algorithm $\mathcal{A}$ that influences the growth - equivalently, as the nodes choose their links ${ }^{1}$ based on the node ranking by $\mathcal{A}$, we say that the system "has adopted" algorithm $\mathcal{A}$. We introduce a model which features three essential elements:

1. Growth. At each time step, one new node enters the system. Nodes can thus be labeled directly by the time step in which they appeared. Each new node creates $m$ directed links to $m$ different preexisting nodes ${ }^{2}$.

2. Ranking-driven attachment. With probability $\beta$, node $t$ chooses its target according to the probability

$$
P^{(\mathcal{A})}(j, t)=\frac{r_{j}(t)^{-\alpha}}{\sum_{l=1}^{t-1} r_{l}(t)^{-\alpha}},
$$

where $r_{j}(t)$ is the ranking position of node $j$ at time $t$ according to $\mathcal{A}$; the real number $\alpha \geq 0$ is a tunable model parameter referred to as exploration cost by Ciampaglia et al.[7]: Large values of $\alpha$ imply that the nodes are only willing to connect to the top-nodes by the adopted ranking algorithm, whereas low values of $\alpha$ allow the nodes to also connect to low-ranked nodes.

\footnotetext{
${ }^{1}$ The network's directed links might be interpreted as friendship or follower relationships in online social networks, or as citations between documents in information networks.

${ }^{2}$ Self-loops and multiple links between a given pair of nodes are prohibited.
} 
3. Quality-driven attachment. With probability $1-\beta$, node $t$ chooses its target according to the probability

$$
P^{(q)}(j, t)=\frac{q_{j}}{\sum_{l=1}^{t-1} q_{l}} .
$$

Our model reduces to the model by Fortunato et al. [12] in the special case $\beta=1$; node quality and quality-driven attachment are novel elements with respect to Fortunato et al.'s model [12]. Differently from the recent popularity dynamics model by Ciampaglia et al. [7] which considers a cultural market composed of a fixed number $N$ of items, our model represents a network that grows with time. Differently from previous works [7, 13, 30], we aim to use our growing network model to compare the long-term properties of the networks generated based on different ranking algorithms.

\subsection{Ranking algorithms}

As our main goal is to uncover the long-term implications of the temporal bias of static centrality metrics and the benefits from suppressing such bias, we focus here on indegree and rescaled indegree [27]. Besides, we introduce a random promotion mechanism which "perturbes" the original ranking by rescaled indegree by promoting randomly-selected nodes to the top of the ranking, and we consider a random ranking of the node as a baseline. We provide below the details of these four ranking algorithms.

1. Ranking by indegree, $k$. The indegree ${ }^{3}$ of a node is defined as the number of incoming connections received by that node [32]. We simply rank the nodes in order of decreasing indegree $k$, which is arguably the simplest way to rank the nodes in a directed network [32]. In growing networks, node indegree is strongly biased by node age $[22,27,33]$, as confirmed by numerical simulations and analytic computations with our model (see Appendix B).

2. Ranking by (age-)rescaled indegree, $R(k)$. We rank the nodes in order of decreasing age-rescaled indegree [27] $R(k)$. The rescaled indegree is built on indegree by requiring that node score is not biased by node age. More specifically, for each node $i$, we consider a reference set $\mathcal{R}_{i}:=\{i-\Delta / 2, \ldots, i+$ $\Delta / 2\}$ of $\Delta+1$ nodes of similar age as node $i$ - we set $\Delta=0.01 N$. We compute the mean $\mu_{i}(k)$ and the standard deviation $\sigma_{i}(k)$ of node indegree within this

\footnotetext{
${ }^{3}$ In the following, we will use interchangeably "indegree", "popularity", and "cumulative popularity". This is because, in our simple setting, the incoming links received by a node are the only available information on its "popularity". The situation might be different in a real online system where, for example, the popularity of a video can be quantified by the number of downloads, by the number of views, by the number of shares, etc.
} 
reference set. In formulas,

$$
\begin{aligned}
\mu_{i}(k) & =\frac{1}{\Delta+1} \sum_{j \in \mathcal{R}_{i}} k_{j} \\
\sigma_{i}(k) & =\sqrt{\frac{1}{\Delta+1} \sum_{j \in \mathcal{R}_{i}}\left(k_{j}-\mu_{i}(k)\right)^{2}}
\end{aligned}
$$

. The rescaled indegree score $R_{i}(k)$ of node $i$ is given by the $z$-score [27]

$$
R_{i}(k)=\frac{k_{i}-\mu_{i}(k)}{\sigma_{i}(k)} .
$$

The rescaled indegree of a given node thus quantifies how larger the node's indegree is with respect to nodes of similar age, in units of standard deviations. Using the $z$-score to normalize static metrics of node importance is customary in scientometrics $[25,53,60]$ where scholars aim to gauge the impact of a given scientific paper independently of its field and publication date [54]. Besides, in citation networks, the rescaled indegree allows us to identify significantly earlier important papers [27, 33], movies [39] and patents [28] with respect to citation count.

3. Ranking by age-rescaled indegree with Random Promotion, $(R(k)+R P)$. While age-rescaled indegree substantially suppresses the cumulative advantage of older nodes [27, 33], it may still amplify the advantage of some nodes that received quickly many connections with respect to nodes of similar age. To reduce this potential problem and further increase diversity, we introduce the ranking by rescaled indegree with random promotion $(R(k)+\mathrm{RP})$ : We rank the nodes by age-rescaled indegree, $R(k)$, and we "promote" $P$ randomly-selected nodes to the top- $T$ of the ranking. Therefore, a fraction $\eta:=P / T$ of nodes in the top- $T$ by the ranking is purely determined by noise. By placing some previously overlooked nodes at the top of the ranking, this mechanism gives these nodes enhanced visibility and, therefore, an additional opportunity to attract some links. In the following, we show results for $T=10, \eta=0.5$; results for other values of $T$ and $\eta$ $(\eta=P / T=5 / 10,8 / 10,10 / 10,10 / 20,16 / 20,20 / 20)$ are shown in the Supplementary Material (Supplementary Figures S11-S13 and S19-S24).

4. Random ranking. The nodes are ranked at random. The resulting ranking is used as a baseline to understand for which parameter values the final indegree-quality correlation benefits from the rankings by indegree and rescaled indegree.

For all the ranking algorithms considered above, if two or more nodes happen to have the same value of the score, their relative order is determined at random. 
Choosing the quality distribution deserves some attention. A simple mean-field approximation shows that for $\beta=0$, the final popularity of the nodes $k$ is expected to be proportional to node quality; simulation results show that for $\beta>0$, node final popularity is a power-law function of node quality (see Appendix B for details). Motivated by this property, to mimic the broad popularity distributions typically observed in real data [32], we choose a Pareto distribution of the quality values $q$ (see Appendix A for details). This choice leads indeed to broad indegree distributions as shown in Fig. S1. We refer to Appendix A for all the simulation details.

We emphasize that the rescaling procedure described above is only one among the possible ways to design a time-aware ranking algorithm [22]. Already in 2005, O'Madadhain and Smyth [35] recognized that static centrality metrics are based on time-aggregate network representations and, for this reason, they do not respect the sequence of events that led to the formation of the network. To solve this issue, O'Madadhain et al. introduced [35] and validated [36] a ranking algorithm based on the detailed contact time-series, acting as precursors of the recent stream of literature on centrality in temporal networks based on time-preserving paths $[22$, $46,47,57]$. We refer to [22] for a review of time-dependent ranking algorithms in complex networks. Nevertheless, we focus on the age-rescaled indegree here because of its simplicity and effectiveness in suppressing the indegree's bias towards old nodes [22, 27]. Testing alternative time-dependent ranking algorithms within our model-generated benchmark graphs is an interesting possibility for future research.

\subsection{Evaluating the algorithms' long-term impact}

To assess the long-term impact of different algorithms, we grow random networks based on the model described above for each ranking algorithm $\mathcal{A}$. The algorithm determines, at any time, the ranking of the nodes that, in turn, determines the probability that a node receives a new connection, according to Eq. (1). We refer to the networks generated with the algorithm $\mathcal{A}$ as to the $\mathcal{A}$-generated networks. Ideally, we would expect a good ranking algorithm $\mathcal{A}$ to exhibit the three main properties introduced above: (i) Quality promotion: The algorithm generates networks where the final popularity of the nodes strongly correlates with their quality; (ii) Quality detection: The algorithm is effective in identifying highquality nodes; (iii) Popularity diversity: The algorithm generates networks where the popularity is not strongly concentrated among few nodes. In the following, we introduce three classes of observables to quantify these three properties.

Quality promotion. For a given ranking algorithm $\mathcal{A}$, we evaluate how well the final popularity $k$ of the nodes reproduces the inherent quality values $q$ for $\mathcal{A}$ generated networks. We calculate the Pearson's linear correlation $r^{\mathcal{A}}(k, q)$ between node popularity $k$ and node quality $q$. While this metric takes all the nodes into account, we are also interested in the algorithm's ability to promote the top-quality 
nodes. To this end, we measure the precision $P_{100}^{\mathcal{A}}(k, q)$ defined as the fraction of nodes that are placed in the top-100 of both the ranking by $k$ and the ranking by $q$. Nevertheless, quality promotion is not sufficient alone to evaluate the metrics because, by construction, the nodes have a non-zero probability to choose their targets based on quality. As a consequence, even the random ranking of the nodes produces networks with non-zero indegree-quality correlation. Such correlation increases as the nodes' sensitivity to quality increases (i.e., as $\beta$ decreases) - see Fig. 2 and the related discussion below. However, the random ranking is useless to find valuable nodes in the system. For this reason, we study not only quality promotion, but also quality detection.

Quality detection. For a given ranking algorithm $\mathcal{A}$, we evaluate how well the scores by $\mathcal{A}$ reproduce the inherent quality values $q$ for $\mathcal{A}$-generated networks. To this end, we measure the Pearson's linear correlation $r^{\mathcal{A}}(s, q)$ between the nodelevel scores $s$ produced by the algorithm $\mathcal{A}$ (measured at the end of the network growth) and node quality $q$ for $\mathcal{A}$-generated networks. In parallel, we also measure the precision [23] $P_{100}^{\mathcal{A}}(s, q)$ of the algorithm, defined as the fraction of nodes that are placed in the top-100 of both the ranking by $s$ and the ranking by $q$.

Diversity. To quantify the ability of the algorithms to evenly spread popularity across the network's nodes, we measure the indegree's Herfindahl index [16] $H(\mathbf{k})$.

$$
H(\mathbf{k})=\sum_{i=1}^{N}\left(\frac{k_{i}}{L}\right)^{2}
$$

where $L$ is the total number of links. The index is proportional to the variance of the network's indegree distribution: the smaller $H(\mathbf{k})$, the less concentrated indegree is within a restricted group of nodes. More specifically, the index ranges between $H_{\min }=1 / N$ (egalitarian network where all the nodes have indegree equal to $L / N$ ) and $H_{\max }=1$ (network where one node has indegree $L$, and all the other nodes have indegree equal to zero). We define $N_{\text {eff }}(\mathbf{k})=1 / H(\mathbf{k})$ as the "effective number of nodes" that received incoming links. Such number $N_{\text {eff }}$ is equal to 1 if one single node received all the incoming links, and it is equal to $N$ if all the nodes received the same amount of links. We posit that a good ranking algorithm should not produce too concentrated networks, and its generated networks should therefore exhibit relatively large values of $N_{\text {eff }}$.

\section{Results}

We grow networks of $N=10,000$ nodes according to the model described above; we refer to Appendix A for all the simulation details. Here, we show the results for node outdegree $m=6$; the results for $m=3$ are commented below 

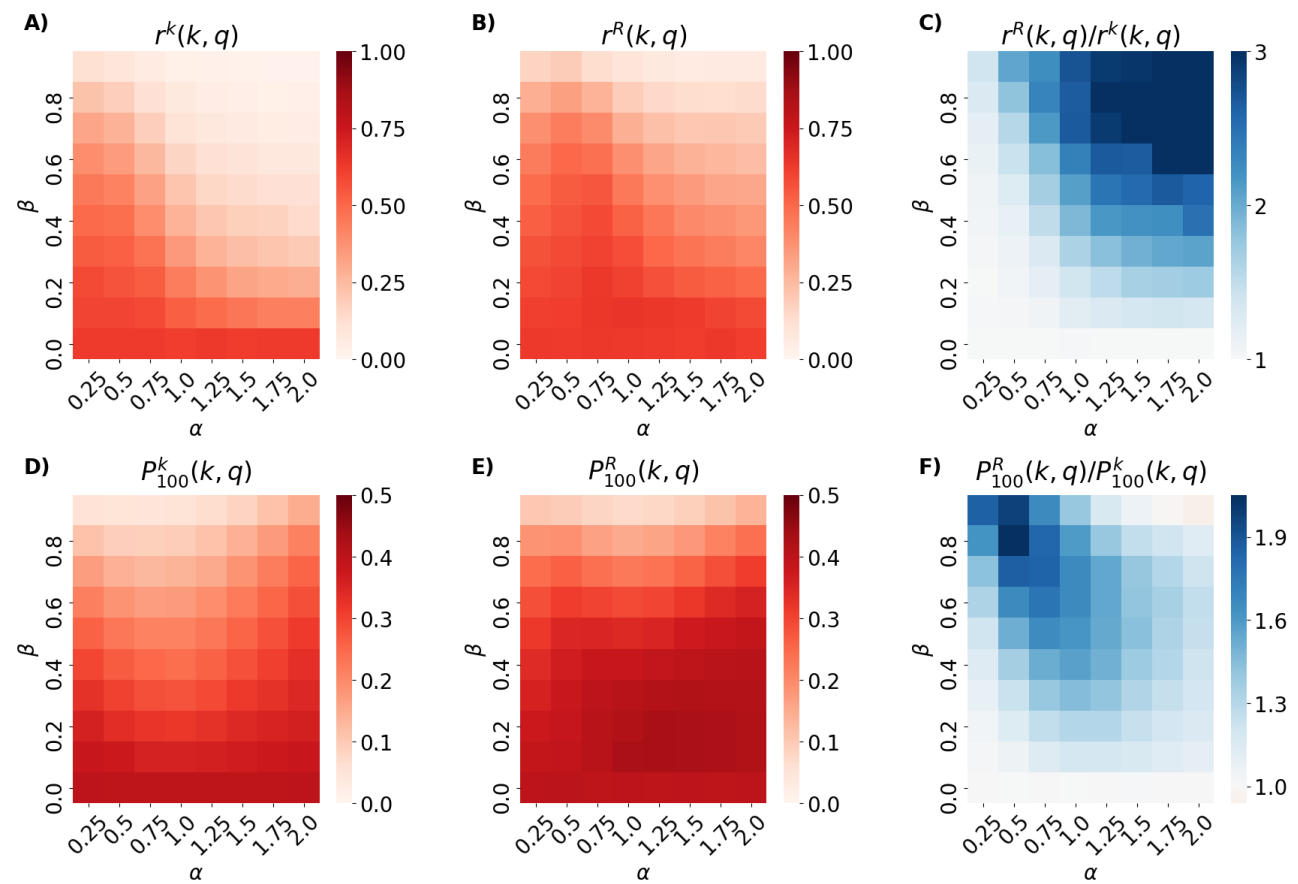

Figure 1: Quality promotion as measured by $r(k, q)$ (the Pearson's linear correlation between node indegree $k$ and node quality $q$-top panels), and $P_{100}(k, q)$ (the precision of node indegree $k$ in identifying the top-100 nodes by quality $q$ - bottom panels): comparison between indegreegenerated and $R(k)$-generated networks. (A-B): $r(k, q)$ for indegree-generated $\left(r^{k}(k, q)\right.$, panel $\mathrm{A})$ and $R(k)$-generated $\left(r^{R}(k, q)\right.$, panel $\left.\mathrm{B}\right)$ networks, as a function of the model parameters $\alpha$ (exploration cost) and $\beta$ (reliance on ranking). (C): Ratio $r^{R}(k, q) / r^{k}(k, q)$ as a function of the model parameters. (D-E): $P_{100}(k, q)$ for indegree-generated $\left(P_{100}^{k}(k, q)\right.$, panel D) and $R(k)$-generated $\left(P_{100}^{R}(k, q)\right.$, panel E) networks, as a function of the model parameters. $(\mathrm{F})$ : Ratio $P_{100}^{R}(k, q) / P_{100}^{k}(k, q)$ as a function of the model parameters. Results are averaged over 500 realizations.

and shown in the SM (Figs. S2-S13). Our goal is to compare the properties of networks generated by the four ranking algorithms defined in Section 2, according to the observables described above.

\subsection{Quality promotion: Impact of the age bias suppression}

Fig. 1A shows the indegree-quality Pearson's linear correlation $r(k, q)$ in indegreegenerated networks, as a function of the model parameters $\alpha$ and $\beta$. The correlation between final popularity and quality is sensitive to both $\alpha$ and $\beta$. As $\alpha$ grows, it becomes harder for low-ranked high-quality nodes to acquire new incoming connections, which results in a lower indegree-quality correlation. The (approximately) monotonous dependence of $r^{k}(k, q)$ on $\alpha$ was not found for the model of a static 

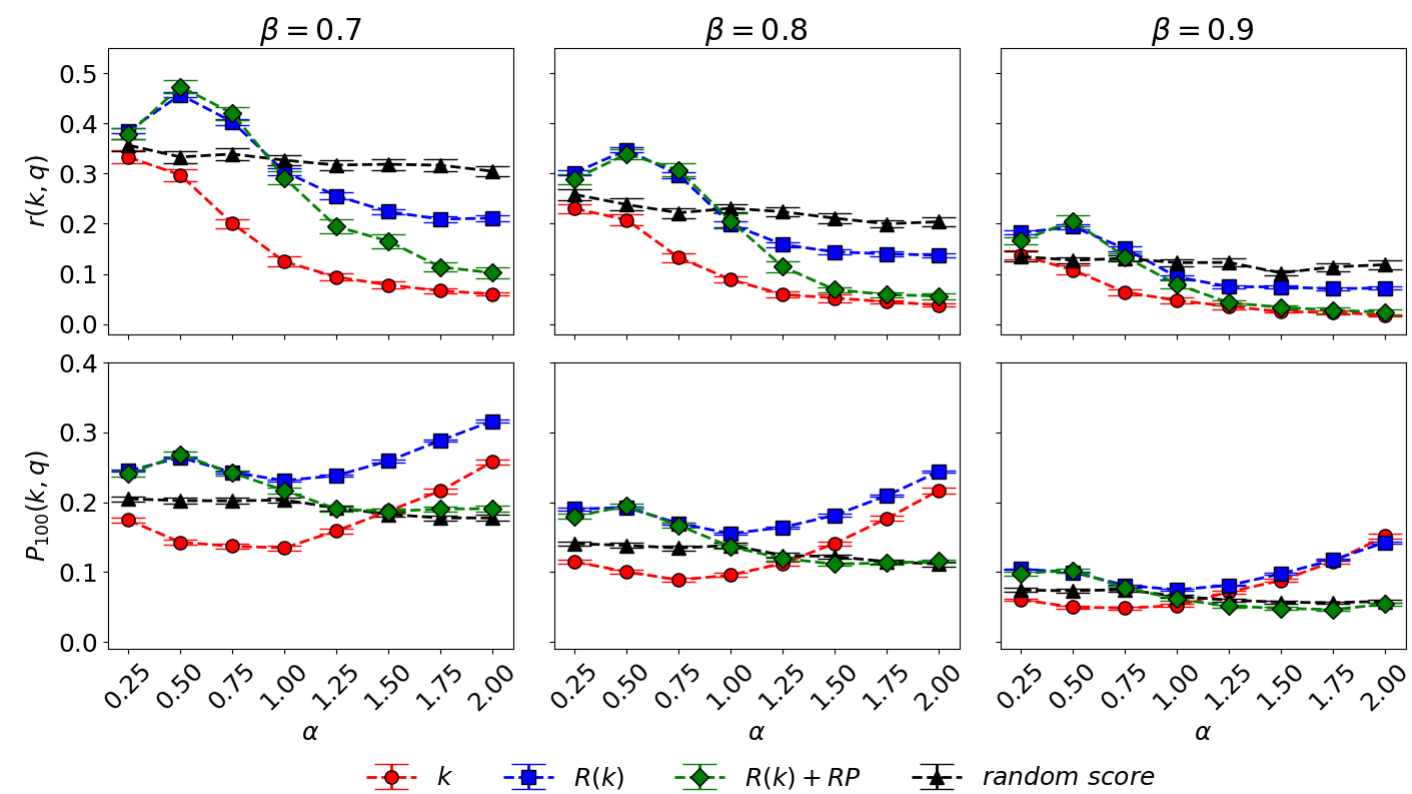

Figure 2: Quality promotion as measured by $r(k, q)$ (the Pearson's linear correlation between node indegree $k$ and node quality $q$ - top panels), and $P_{100}(k, q)$ (the precision of node indegree $k$ in identifying the top-100 nodes by quality $q$ - bottom panels): comparison between indegreegenerated (red circles), $R(k)$-generated (blue squares), $R(k)+R P$-generated (green rhombuses), and random-generated networks (triangles). The three columns correspond, from left to right, to $\beta=0.7,0.8,0.9$, respectively. Results are averaged over 100 realizations; the error bars represent the standard error of the mean.

market ${ }^{4}$ by Ciampaglia et al. [7], which indicates that it is a consequence of the network's growth. As $\beta$ grows, the nodes become less sensitive to quality and, as a direct consequence, the indegree-quality correlations deteriorates.

Fig. 1B shows the correlation $r^{R}(k, q)$ between node indegree $k$ and node quality in $R(k)$-generated networks. The figure shows that by adopting the age-rescaled metric $R(k)$, the indegree-quality correlation stays large for a broader parameter region. For example, we observe values of $r^{R}(k, q)$ as high ${ }^{5}$ as 0.35 when $(\alpha, \beta)=$ $(1,0.7)$, which corresponds to a scenario where the nodes are driven by quality only three times out of ten.

To visually appreciate the parameter regions where the indegree-quality correlations significantly differ among the two classes of networks, we represent the heatmap of the ratio $r^{R}(k, q) / r^{k}(k, q)$ (Fig. $\left.1 \mathrm{C}\right)$. When $\beta=0$, all the nodes are

\footnotetext{
${ }^{4} \mathrm{By}$ static market, we mean a collection of a fixed number of items. This is different from our growing network model where, at each time step, a new node enters the system and connects to the preexisting nodes.

${ }^{5}$ As opposed to $r^{k}(k, q)=0.12$ observed for indegree-generated networks for the same pair of $(\alpha, \beta)$ values.
} 
only sensitive to quality, and the plotted ratio is thus one by definition (on average) because node ranking has no influence. As soon as $\beta>0$, the nodes become driven both by quality and by ranking, which makes it possible to reveal the differences between the networks grown with different algorithms. We find that rescaled indegree produces networks with a higher indegree-quality correlation for all the parameter space; we observe the largest advantage of the rescaled indegree in terms of quality promotion for the region where both $\alpha$ and $\beta$ are relatively large - i.e., in the region where the nodes are unwilling to choose low-ranked nodes and, at the same time, are highly sensitive to ranking. Analogous heatmaps for the precision metrics (Figs. 1D-F) show that the indegree's precision in $R(k)$-generated networks is systematically larger than that in indegree-generated networks. Differently from Fig. 1C, Fig. 1F shows that the largest gaps between the precision in $R(k)$ - and indegree-generated networks occur in the small $\alpha$, large $\beta$ region. We discuss the reasons behind the different trend for correlation and precision in the next paragraph.

\subsection{Quality promotion: comparing the four ranking algorithms}

Fig. 1 indicates that adopting the rescaled indegree allows us to better promote node quality for a broad range of model parameters. At the same time, it is important to compare its performance with that observed in networks generated with both the ranking by rescaled indegree with random promotion, and the random algorithm. As pointed out above, for $\beta<1$, the nodes have a non-zero probability to choose their targets based on quality, which results in a non-zero indegree-quality even for networks generated with a random ranking. We focus on three values of $\beta$ $(\beta=0.7,0.8,0.9)$ which correspond to populations of nodes that are mostly driven by ranking when selecting their targets; analogous results for smaller values of $\beta$ are shown in Supplementary Figs. S16-S18 for $m=6$ and S8-S10 for $m=3$.

We find (Fig. 2, top panels) that the indegree-quality correlation observed in $R(k)$-generated networks is not always larger than the indegree-quality correlation observed in random-generated networks: as the exploration cost $\alpha$ grows, the indegree-quality correlation in $R(k)$-generated networks dwindles; when $\alpha$ is larger than one, $R(k)$-generated networks exhibit a smaller indegree-quality correlation than random-generated networks. While a large exploration cost is harmful for the overall indegree-quality correlation in both indegree-generated and $R(k)$-generated networks, for $\alpha \geq 1$, indegree's precision in promoting the top-quality nodes (Fig. 2 , bottom panels) tends to grow with the exploration cost. Remarkably, $R(k)$ generated networks exhibit the largest precision values for all the values of $\alpha$.

The behavior of the $(R(k)+\mathrm{RP})$-generated networks (i.e, the networks generated with the ranking by rescaled indegree with random promotion, $R(k)+\mathrm{RP})$ is non-trivial. Indeed, for small values of $\alpha$, the indegree-quality correlation and indegree's precision of these networks are comparable with those of $R(k)$-generated 
networks, which indicates that randomly promoting a few nodes to the top of the ranking is not harmful to quality promotion. On the other hand, for $\alpha>1$, the indegree-quality correlation (or indegree's precision) of $R(k)$-generated networks becomes larger than that of $(R(k)+\mathrm{RP})$-generated networks. We conclude that for large values of $\beta(\beta=0.7,0.8,0.9)$, the random promotion mechanism has a marginal impact on quality promotion for sparse networks when $\alpha<1$, whereas it is harmful to quality promotion when the exploration cost is large, i.e., for $\alpha>1$. For smaller values of $\beta(\beta=0.1,0.3,0.5)$, the $(R(k)+\mathrm{RP})$-generated networks and the $R(k)$-generated networks exhibit comparable values of indegree-quality correlation and precision (Figs. S8-S16).

The qualitative difference between the top and the bottom panels of Fig. 2 is explained by the different indegree distributions of the networks generated with different values of $\alpha$. When $\alpha$ is large, the incoming links are concentrated on few top items (as the effective number of nodes shows, see Fig. 4 below and the related discussion) and, at the same time, the low-quality items remain unnoticed. The small number of incoming links received by low-quality items do not allow the metrics to discriminate their quality, which results in small indegree-quality correlation values. By contrast, high-quality nodes receive a large number of incoming links, and it is possible for the metrics to rank them at the top, which results in relatively large precision values.

\subsection{Quality detection}

In indegree-generated and $R(k)$ - generated networks, nodes' indegree and agerescaled indegree, respectively, are the scores that are used for the nodes' ranking. Our ability to detect quality in such networks is determined by the strength of the relation between node score $s$ and $q$ (as measured by both the Pearson's linear correlation $r^{s}(s, q)$ and the precision $\left.P_{100}^{s}(s, q)\right)$. Remarkably, the correlation $r^{R}(R(k), q)$ is larger than the correlation $r^{k}(k, q)$ for all the parameter values (Fig. 3, top panels, and Fig. S14). The precision of age-rescaled indegree in identifying the top-quality nodes is also larger than indegree's precision for all the parameter values (Fig. 3, bottom panels). In qualitative agreement with the results obtained for quality promotion (Fig. 1), for $m=6$ and $\beta=0.7,0.8,0.9$, the random promotion mechanism turns out to have a marginal impact for $\alpha<1$, whereas it is harmful for $\alpha>1$. For $m=3,6$ and $\beta=0.1,0.3,0.5$, the $(R(k)+\mathrm{RP})$-generated and $R(k)$-generated networks exhibit similar levels of score-quality correlation (see Figs. S9 and S17).

By being completely insensitive to node popularity, the random score always achieves zero precision, on average, in identifying the top-quality nodes. As expected, while the random score can still generate networks with non-zero indegreequality correlation (Fig. 2), the rankings it produces have no practical utility. 

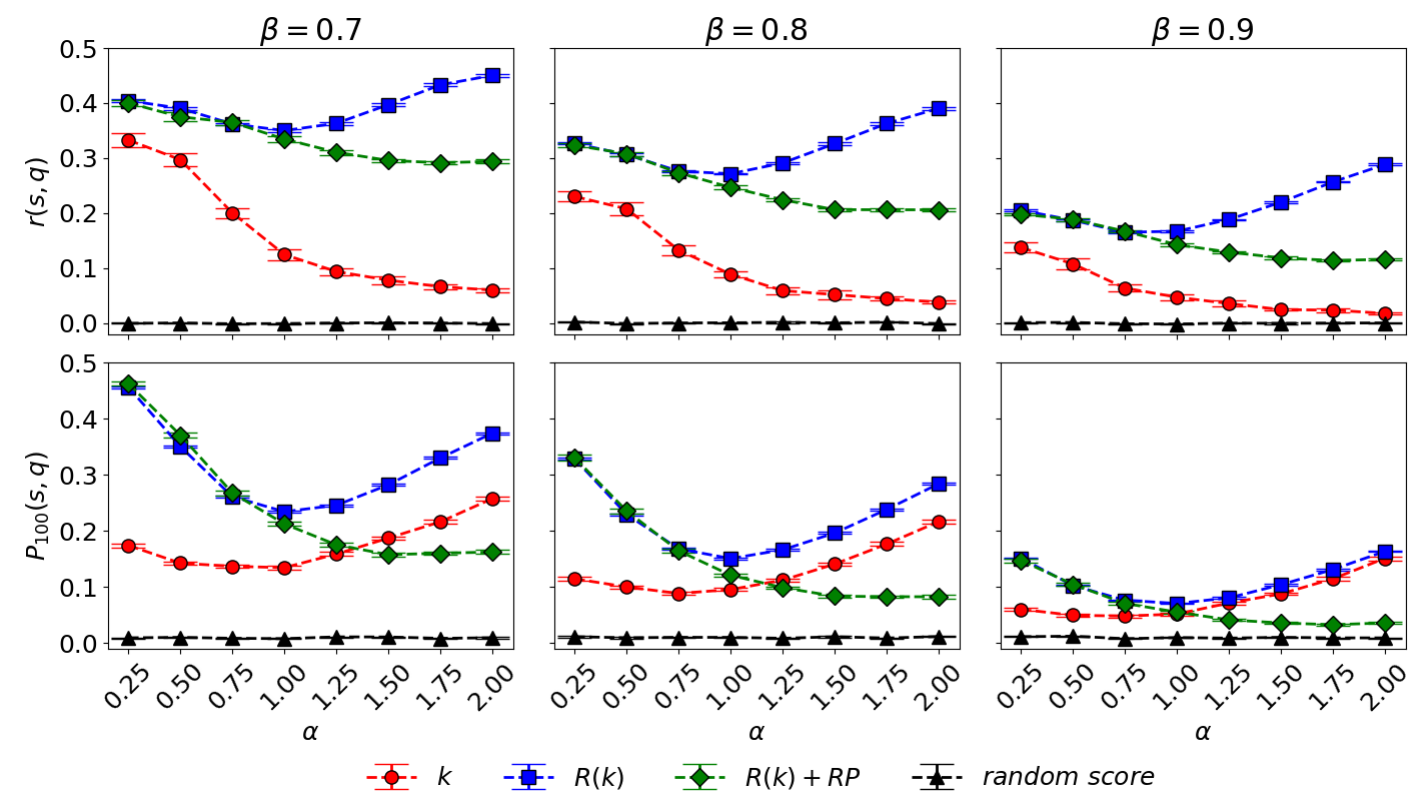

$R(k)$

- 드 $R(k)+R P$

random score

Figure 3: Quality detection as measured by $r(s, q)$ (the Pearson's linear correlation between node score $s$ and node quality $q$-top panels), and $P_{100}(s, q)$ (the precision of node score $s$ in identifying the top-100 nodes by quality $q$ - bottom panels): comparison between indegree-generated $(s=k$, red circles), $R(k)$-generated ( $s=R(k)$, blue squares), $(R(k)+R P)$-generated ( $s=R(k)$, green rhombuses), and random-generated networks ( $s$ is given by a random score, black triangles). The three columns correspond, from left to right, to $\beta=0.7,0.8,0.9$, respectively. The dots represent averages over 100 realizations; the error bars represent the standard error of the mean.

\subsection{Diversity}

For both indegree- and $R(k)$-generated networks, the effective number of nodes $N_{\text {eff }}$ depends on both $\alpha$ and $\beta$ (Figs. 4). Unsurprisingly, the random score produces the most egalitarian networks (Fig. 4), with $N_{\text {eff }}$ values above $3000=0.3 N$. In qualitative agreement with previous findings [43], the net effect of a larger sensitivity to the nodes' ranking position is, for all the studied metrics, a more unequal popularity distribution, which manifests itself in the decrease of $N_{\text {eff }}$ as $\alpha$ increases. Importantly, the popularity distribution is evener for $R(k)$-generated networks than for indegree-generated networks (Figs. 4 and S15), and the $(R(k)+\mathrm{RP})$ algorithm further enhances popularity diversity. In summary, the age normalization procedure not only improves the indegree-quality and the score-quality correlation, but it also decreases the popularity inequality in the system; as expected, the random promotion mechanism further decreases the popularity inequality. At the same time, both the $R(k)$-generated and the $(R(k)+\mathrm{RP})$-generated networks exhibit $N_{\text {eff }}$ values significantly smaller than the $N_{\text {eff }}$ achieved by the random ranking. It remains open to design ranking algorithms that lead to more egalitarian networks than those generated with rescaled indegree and $(R(k)+\mathrm{RP})$, yet maintaining a 

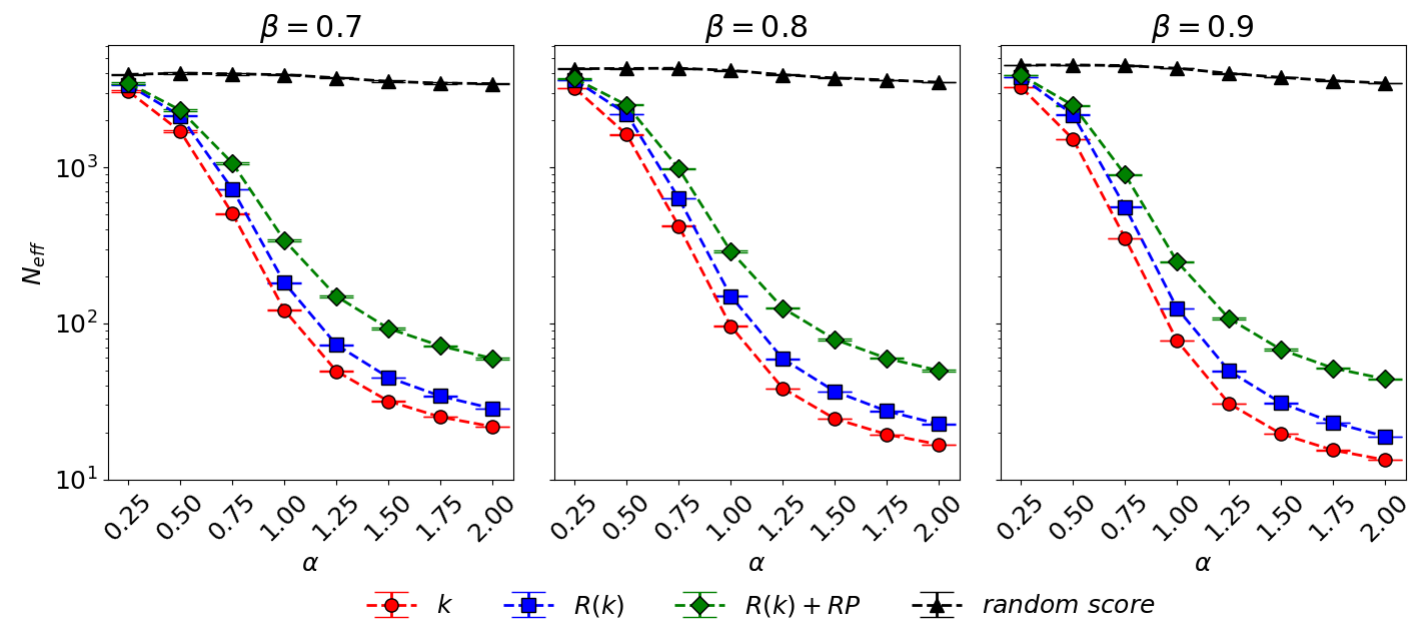

Figure 4: Diversity as measured by $N_{\text {eff }}$ (the larger, the more egalitarian the indegree distribution): comparison between indegree-generated (red circles), $R(k)$-generated (blue squares), $(R(k)+R P)$-generated (green rhombuses) and random-generated (black triangles) networks. The three columns correspond, from left to right, to $\beta=0.7,0.8,0.9$, respectively. The dots represent averages over 100 realizations; the error bars represent the standard error of the mean.

similar level of quality promotion and quality detection.

\subsection{Including node removal in the model}

In real social and information systems, not only new nodes can enter the system, but also existing nodes can disappear or lose relevance. The members of an online community, for example, may lose interest in the platform and deactivate their account [10], which may eventually lead to the "death" of the platform [14]. In the WWW, many webpages are deleted every day, which makes it essential to incorporate node deletion into growing network models [20]. To take into account this situation, we introduce a variant of the model introduced in Section 2.1 where the nodes are in one of two possible states: "active" and "removed". At every time step $t$, each active node becomes removed with probability $\mu$, and one new active node enters the system and chooses its targets among the existing active nodes according to the rules described in Section 2.1. In the continuum approximation, the number of active nodes, $A(t)$, follows the equation $\dot{A}(t)=1-\mu A(t)$ whose solution (with the initial condition $A(1)=1$ ) is

$$
A(t)=\mu^{-1}+\left(1-\mu^{-1}\right) \exp (-\mu(t-1))
$$

After an initial linear growth $\left(A(t) \approx t\right.$ for $\left.t \ll \mu^{-1}\right)$, the number of active nodes eventually equilibrates at $A^{*}=1 / \mu$. The validity of Eq. (5) is confirmed by our numerical simulations (see Fig. $5 \mathrm{~F}$ ). Note that when $\mu=0$ (i.e., in the model without node removal), $A(t)=t$ and $N=t^{*}$, where $t^{*}$ denotes the total number 
of performed simulation steps. In the following, we set $t^{*}=10^{4}$; due to the node removal mechanism, the number $A\left(10^{4}\right)$ of active nodes at the end of the simulation is substantially smaller than $10^{4}$, as expected based on Eq. (5) (see Fig. 5F).

We investigated whether this node removal mechanism alters substantially the results described above. More specifically, we performed numerical simulations with values of $\mu$ ranging from $10^{-4}$ to $5 \cdot 10^{-4}$, which corresponds to expected values of the asymptotic number of active nodes, $A^{*}=1 / \mu$, ranging from 2,000 to 10,000 . We find that through the whole range of $\mu$ values, the results are qualitatively similar to those obtained for the networks without node removal (see Figs. 5A-E for the results for $\alpha=0.5, \beta=0.7$, and Figs. S25 for $\alpha=1, \beta=$ 0.7). All the considered observables display a remarkable stability with respect to $\mu$, which indicates that node removal has a marginal impact on the algorithms' quality promotion, quality detection, and popularity diversity. It remains open to determine the largest removal probability $\mu$ tolerated by the system before the results change qualitatively.

\subsection{A case study: growing a network of scientific papers based on different metrics}

So far, we have considered model-generated networks. How are our model and our results on ranking algorithms relevant to real systems? If our model provides a plausible description of the growth of a given system of interest, and we have a reliable way to infer the $(\alpha, \beta)$ parameters from the available data, we would be able to quantify the potential impact of various ranking algorithms on the future properties of the system. Stimulated by this observation, in this Section, we analyze real information networks to fit our model's parameter to the empirical data, and use the resulting parameters to grow again the network based on different ranking algorithms.

The information networks analyzed here are subsets of the American Physical Society (APS) citation network of scientific papers ${ }^{6}$. The citation dataset provided by the APS contains all 539974 papers published by the APS from 1893 to 2013 together with their 5992897 references to other papers published by APS journals and their publication date. For our analysis, we extract two subsets of papers that include the papers with the PACS number" 89.75.Hc ("Networks and genealogical trees"; the subset includes $N=1615$ papers and $L=8222$ citations) and 03.67.Lx ("Quantum computation architectures and implementations"; the subset includes $N=3876$ papers and $L=24213$ citations), respectively.

\footnotetext{
${ }^{6}$ The dataset has been already analyzed in $[27,28,30]$, and it can be downloaded here: https: //journals.aps.org/datasets.

${ }^{7}$ The PACS (Physics and Astronomy Classification Scheme) codes refer to a hierarchical classification of research areas in physics, astronomy, and related sciences. We refer to https: //journals.aps.org/PACS for further information.
} 
A)

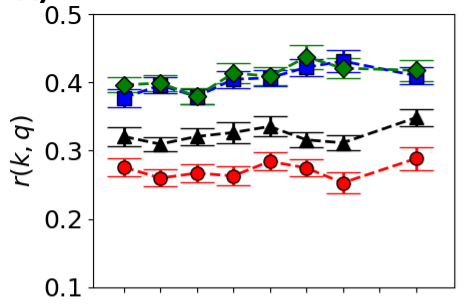

D)

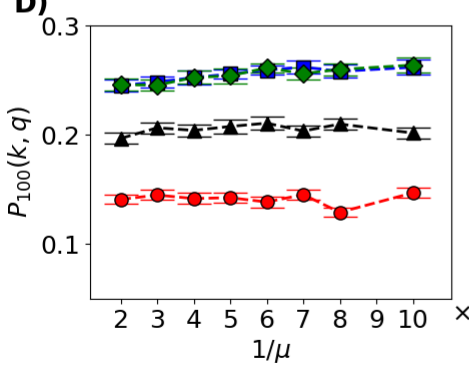

B)

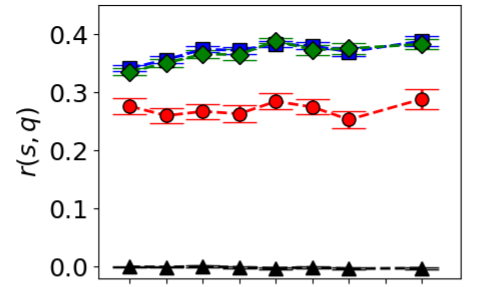

E)

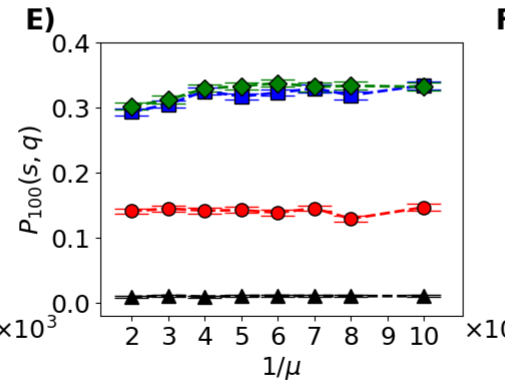

C)

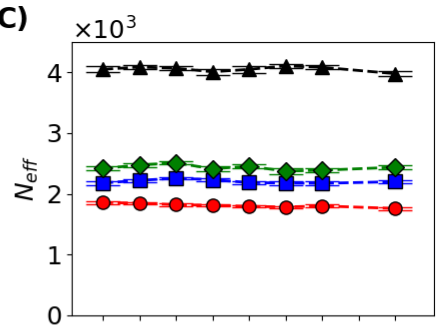

F)

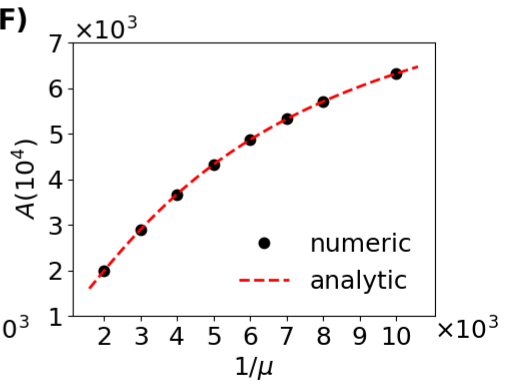

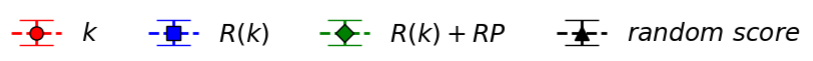

Figure 5: The impact of node removal on quality promotion, quality detection, popularity diversity. We grow networks generated with the model described in Section 3.5 with $\alpha=0.5, \beta=0.7$, $T=10^{-4}$. We show five ranking evaluation metrics as a function of the inverse of the removal probability, $A^{*}=\mu^{-1}$ : (A) the Pearson's correlation between node indegree and node quality; (B) the Pearson's correlation between node score and node quality (see Fig. 3's caption for the definition of node score); (C) the effective number of nodes, $N_{\text {eff }}$; (D) the indegree's precision in identifying the top-100 nodes by quality; (E) the score's precision in identifying the top-100 nodes by quality. As in the previous figures, different lines correspond to the networks generated with different algorithms. The symbols represent averages over 50 realizations; the error bars represent the standard error of the mean. Panel $\mathrm{F}$ shows the number of active nodes at time $t=10^{4}$, i.e., at the time when we halt the simulations, as a function of $\mu^{-1}$; the values computed analytically through Eq. (5) well match the values observed in the simulations.

To quantify the potential impact of different ranking algorithms, the first step is to infer the optimal pair $(\hat{\alpha}, \hat{\beta})$ of model parameters together with the optimal algorithm that together lead to the best agreement between the $\mathcal{A}$-generated model networks and the observed real network. The real data determine the final number of nodes in the network, their order of appearance, and their outdegree values; in an $\mathcal{A}$-generated model network, the nodes choose their references based on the rules of the model described in Section 2.1, where the ranking position of the nodes is determined by the chosen algorithm $\mathcal{A}$.

Quality is not accessible in real data as opposed to synthetic networks where it is a well-defined node-level variable. To overcome this limitation, as rescaled indegree is the best-performing metric in quantifying node quality in synthetic networks (Fig. 3), we use the papers' final rescaled indegree score $R(k)$ as a proxy for their quality if $R(k) \geq 0$, whereas we set $q=0$ for papers such that $R(k)<0$. 
The agreement between the $\mathcal{A}$-generated networks and the original network is quantified by the model's mean error per node, $e^{(\mathcal{A}, \alpha, \beta)}$. For each $\mathcal{A}$-generated network $^{8} \mathcal{G}(\mathcal{A}, \alpha, \beta)$, we first define the network's mean error per node, $e(\mathcal{G}(\mathcal{A}, \alpha, \beta))$, as

$$
e(\mathcal{G}(\mathcal{A}, \alpha, \beta))=\frac{1}{N} \sum_{i=1}^{N}\left|k_{i}(\mathcal{G}(\mathcal{A}, \alpha, \beta))-k_{i}^{*}\right|,
$$

where $k_{i}(\mathcal{G})$ denotes paper $i$ 's indegree in network $\mathcal{G}$, whereas $k_{i}^{*}$ denotes the observed indegree of paper $i$. The model's mean error per node, $e^{(\mathcal{A}, \alpha, \beta)}$ is defined as the average of $e(\mathcal{G})$ over a sample of $100 \mathcal{A}$-generated networks. Essentially, the model's mean error per node quantifies the average deviation between node indegree in real and model networks. The lower $e^{(\mathcal{A}, \alpha, \beta)}$, the better the agreement between the $\mathcal{A}$-generated model networks and the real network.

Finally, we compare the properties of the networks generated by different ranking algorithms for $(\alpha, \beta)=(\hat{\alpha}, \hat{\beta})$. The rationale behind such a comparison is that the parameter values $(\hat{\alpha}, \hat{\beta})$ that yield the best agreement represent our best estimations of the nodes exploration cost $\alpha$ and sensitivity to popularity $\beta$; based on this assumption, we estimate the long-term implications of various algorithms by simply changing the algorithm that is used to grow the network, whilst keeping $\alpha=\hat{\alpha}$ and $\beta=\hat{\beta}$ fixed.

For the subset that corresponds to the PACS code 89.75.Hc, we find that indegree-generated networks with $\hat{\alpha}=0.5$ and $\hat{\beta}=0.5$ exhibit the best agreement with the real network (mean error per node $e^{(k, 0.5,0.5)}=3.058$, see Fig. (see Fig. 6A)). Therefore, we fix $\alpha=0.5$ and $\beta=0.5$, and we compare the networks generated by three different algorithms: indegree, age-rescaled indegree, and the ranking by rescaled indegree with random promotion. In qualitative agreement with our previous results, we find that $R(k)$-generated networks and $(R(k)+\mathrm{RP})$-generated networks exhibit substantially larger popularity diversity than $k$-generated networks and the original network (see Fig. 6B). Qualitatively similar results are obtained for the subset that corresponds to the PACS code 03.67.Lx (see Figs. 6CD). These results confirm that in a real information system, suppressing the age bias of popularity metrics can increase popularity diversity and reduce the mean age of the most popular nodes in the system. Beyond numerical simulations, we envision that future studies might further validate this conclusion by means of field experiments where subjects in different groups can select various information items based on the items' ranking position by different ranking algorithms.

\footnotetext{
${ }^{8} \mathrm{An} \mathcal{A}$-generated network is a realization of the stochastic process that generates $\mathcal{A}$-generated networks.
} 
PACS: 89.75.HC


PACS: 03.67.LX
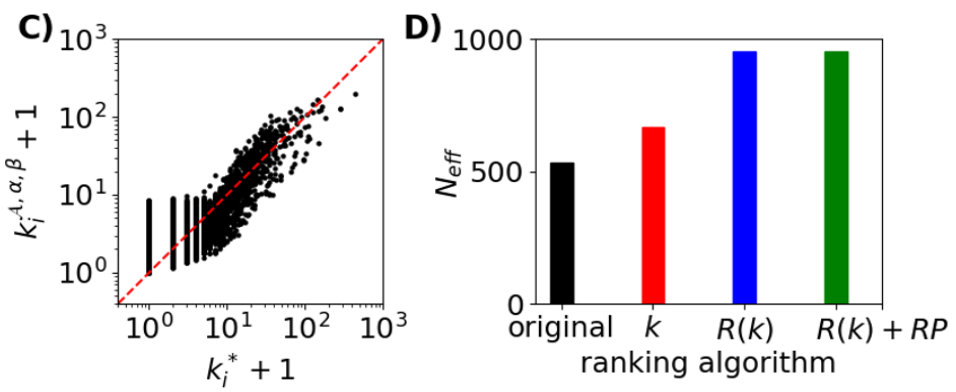

Figure 6: A comparison of two real networks (subsets of the APS citation network) with the respective synthetic networks generated with the model described in Section 2.1, using different ranking algorithms. Panels (A,C) illustrate the scatter plots between the nodes' original indegree $k^{*}$ and the nodes' average indegree $\bar{k}$ in indegree-generated networks $(\alpha=\hat{\alpha}, \beta=\hat{\beta})$ generated as described in the main text, for the APS network's subset that corresponds to the PACS code 89.75.Hc and 03.67.Lx, respectively. Panels (B,D) show the averages of $N_{\text {eff }}$ over 100 realizations of the model networks generated by different ranking algorithms. The $(R(k)+\mathrm{RP})-$ generated $(\eta=0.5, T=10)$ and $R(k)$-generated networks exhibit significantly larger values of $N_{\text {eff }}$ than the original network and the indegree-generated network. This indicates that in a real information system, suppressing the age-bias of popularity-based metrics can substantially improve popularity diversity.

\section{Discussion}

To summarize, we find that age-rescaled indegree allow us not only to fairly compare old and recent nodes [27, 33], but also to produce networks where the nodes' final popularity is better correlated with their quality than for the networks that adopted indegree, and the popularity distribution is more even. Examples of widely-used cumulative popularity metrics include the number of views or downloads for online content, the number of received citations for scientific papers, among others. Our results indicate that despite the widespread use of cumulative popularity metrics, age-rescaled metrics may better help both users to find high-quality content, and high-quality content to experience larger success than low-quality content. Besides, the random promotion mechanism introduced here consistently improves popularity diversity, whereas its impact on quality promotion 
and detection ranges from marginal to substantial depending on the exploration cost parameter $\alpha$.

The main message of our work is that network-based growth models can help us not only to understand the impact of network growth mechanisms on the rankings by a given algorithm $[22,26,29]$, but also to estimate the impact of the adoption of different ranking algorithms by a given system. In other words, we can investigate not only how the past evolution of the system influenced the current rankings, but also how the adopted rankings may influence the future evolution of the system. As a result, our ranking-driven and quality-driven growing network model can be interpreted as a generative model for benchmark graphs to evaluate the performance of ranking algorithms in terms of quality promotion, quality detection, and popularity diversity.

We stress that the ranking algorithms considered here are simple in the sense of being based on a single criterion that is furthermore readily quantified; consider the ranking of nodes by their indegree, for example. The newly proposed ranking algorithm with random promotion is similar as it only requires choosing the original metric and the number of promoted nodes in the top of the ranking. In a general case where multiple criteria are to be considered, especially when they are contradicting such as node popularity and novelty, the existing vast body of literature on multiple-criteria decision analysis $[15,52]$ with techniques such as the analytic hierarchy process [41] and outranking [40] becomes relevant. This goes beyond directly combining node scores by various metrics $[34,61]$ that is often used in the field of network analysis, yet it can become relevant when designing a system for real users with their heterogeneous, and often contradictory, needs and preferences.

Our work sheds light on the long-studied relation between quality/talent and success: Do the high-quality nodes experience larger success than the low-quality nodes? Why nodes of similar worthiness experience widely different success? In real systems, addressing these questions is challenging as defining "node quality" in an unbiased and objective way is often not possible. Our model-based approach bypasses this obstacle by defining node quality as an intrinsic node property, and by building multiple independent realizations of an artificial system where the nodes choose their connections based on both the other nodes' ranking and their quality. At the same time, while the model studied here is arguably one of the simplest models which feature all the elements of interest in our analysis (network growth, and the joint influence of ranking and quality on network growth), it can only provide a stylized description of the growth of real networks.

Finally, the application of our growing network model to the American Physical Society citation network of scientific papers constitutes an attempt to estimate the potential consequences of various ranking algorithms on a given information system. We envision that more sophisticated models together with suitable field 
experiments will improve the reliability of model-based predictions of the effects of ranking algorithms, providing us with a robust basis for more informed choices of ranking algorithms for real-world applications. To draw a parallel, in a similar way as high-resolution models of epidemic spreading have led to accurate predictions of the properties of disease outbreaks [51], detailed models of network evolution may lead to the accurate quantification of the consequences of the adoption of a given metric in a given system.

\section{Acknowledgments}

We thank Yi-Cheng Zhang for many enlightening discussions on the topic. This work has been supported by the National Natural Science Foundation of China (Grants Nos. 61673150, 11622538), the Science Strength Promotion Program of the UESTC, and the Zhejiang Provincial Natural Science Foundation of China (Grant no. LR16A050001). MSM acknowledges the University of Zürich for support through the URPP Social Networks.

\section{Author contributions statement}

M.S.M. and M.M. conceived the idea, M.S.M. and L.L. designed research, S.Z. performed the numerical simulations, S.Z. and M.S.M. performed the analytic computations, all authors analyzed and discussed the results. S.Z. and M.S.M. wrote the manuscript. All authors reviewed the manuscript.

\section{References}

[1] Paolo Boldi and Sebastiano Vigna. Axioms for centrality. Internet Mathematics, 10(3-4):222-262, 2014.

[2] Paolo Boldi, Alessandro Luongo, and Sebastiano Vigna. Rank monotonicity in centrality measures. Network Science, 5(4):529-550, 2017.

[3] Engin Bozdag. Bias in algorithmic filtering and personalization. Ethics and information technology, 15(3):209-227, 2013.

[4] Sergey Brin and Lawrence Page. The anatomy of a large-scale hypertextual web search engine. Computer Networks and ISDN Systems, 30(1):107-117, 1998.

[5] Hongbin Cai, Yuyu Chen, and Hanming Fang. Observational learning: Evidence from a randomized natural field experiment. The American Economic Review, 99(3):864-882, 2009. 
[6] Junghoo Cho and Sourashis Roy. Impact of search engines on page popularity. In Proceedings of the 13th International Conference on World Wide Web, pages 20-29. ACM, 2004.

[7] Giovanni Luca Ciampaglia, Azadeh Nematzadeh, Filippo Menczer, and Alessandro Flammini. How algorithmic popularity bias hinders or promotes quality. Scientific Reports, 8, 2018.

[8] Iain D Couzin, Christos C Ioannou, Güven Demirel, Thilo Gross, Colin J Torney, Andrew Hartnett, Larissa Conradt, Simon A Levin, and Naomi E Leonard. Uninformed individuals promote democratic consensus in animal groups. Science, 334(6062):1578-1580, 2011.

[9] Alejandro Diaz. Through the google goggles: Sociopolitical bias in search engine design. Web search, pages 11-34, 2008.

[10] Gideon Dror, Dan Pelleg, Oleg Rokhlenko, and Idan Szpektor. Churn prediction in new users of yahoo! answers. In Proceedings of the 21st International Conference on World Wide Web, pages 829-834. ACM, 2012.

[11] Robert Epstein and Ronald E Robertson. The search engine manipulation effect (seme) and its possible impact on the outcomes of elections. Proceedings of the National Academy of Sciences, 112(33):E4512-E4521, 2015.

[12] Santo Fortunato, Alessandro Flammini, and Filippo Menczer. Scale-free network growth by ranking. Physical Review Letters, 96(21):218701, 2006.

[13] Santo Fortunato, Alessandro Flammini, Filippo Menczer, and Alessandro Vespignani. Topical interests and the mitigation of search engine bias. Proceedings of the National Academy of Sciences, 103(34):12684-12689, 2006.

[14] David Garcia, Pavlin Mavrodiev, and Frank Schweitzer. Social resilience in online communities: The autopsy of friendster. In Proceedings of the First ACM Conference on Online Social Networks, pages 39-50. ACM, 2013.

[15] Salvatore Greco, J Figueira, and M Ehrgott. Multiple criteria decision analysis. Springer, 2016.

[16] Orris Clemens Herfindahl. Copper costs and prices: 1870-195\%. Baltimore, Published, P, 1959. Published for Resources for the Future by Johns Hopkins Press, 1959.

[17] Matthew Hindman, Kostas Tsioutsiouliklis, and Judy A Johnson. Googlearchy: How a few heavily-linked sites dominate politics on the web. In In Annual Meeting of the Midwest Political Science Association, 2003. 
[18] Dietmar Jannach and Kolja Hegelich. A case study on the effectiveness of recommendations in the mobile internet. In Proceedings of the Third ACM Conference on Recommender Systems, pages 205-208. ACM, 2009.

[19] Jun-Jie Jiang, Zi-Gang Huang, Liang Huang, Huan Liu, and Ying-Cheng Lai. Directed dynamical influence is more detectable with noise. Scientific Reports, 6:24088, 2016.

[20] Joseph S Kong, Nima Sarshar, and Vwani P Roychowdhury. Experience versus talent shapes the structure of the web. Proceedings of the National Academy of Sciences, 105(37):13724-13729, 2008.

[21] Steve Lawrence and C Lee Giles. Accessibility of information on the web. Nature, 400(6740):107-107, 1999.

[22] Hao Liao, Manuel Sebastian Mariani, Matus Medo, Yi-Cheng Zhang, and Ming-Yang Zhou. Ranking in evolving complex networks. Physics Reports, 689:1-54, 2017.

[23] Linyuan Lü, Matúš Medo, Chi Ho Yeung, Yi-Cheng Zhang, Zi-Ke Zhang, and Tao Zhou. Recommender systems. Physics Reports, 519(1):1-49, 2012.

[24] Linyuan Lü, Duanbing Chen, Xiao-Long Ren, Qian-Ming Zhang, Yi-Cheng Zhang, and Tao Zhou. Vital nodes identification in complex networks. Physics Reports, 650:1-63, 2016.

[25] Jonas Lundberg. Lifting the crowncitation z-score. Journal of Informetrics, 1 (2):145-154, 2007.

[26] Manuel Sebastian Mariani, Matúš Medo, and Yi-Cheng Zhang. Ranking nodes in growing networks: When pagerank fails. Scientific Reports, 5, 2015.

[27] Manuel Sebastian Mariani, Matúš Medo, and Yi-Cheng Zhang. Identification of milestone papers through time-balanced network centrality. Journal of Informetrics, 10(4):1207-1223, 2016.

[28] Manuel Sebastian Mariani, Matúš Medo, and François Lafond. Early identification of important patents: Design and validation of citation network metrics. Technological Forecasting and Social Change, 2018.

[29] Matúš Medo and Giulio Cimini. Model-based evaluation of scientific impact indicators. Physical Review E, 94(3):032312, 2016.

[30] Matúš Medo, Giulio Cimini, and Stanislao Gualdi. Temporal effects in the growth of networks. Physical Review Letters, 107(23):238701, 2011. 
[31] Flaviano Morone and Hernán A Makse. Influence maximization in complex networks through optimal percolation. Nature, 524(7563):65, 2015.

[32] Mark Newman. Networks: An introduction. Oxford University Press, 2010.

[33] MEJ Newman. The first-mover advantage in scientific publication. Europhys. Lett., 86(6):68001, 2009.

[34] Kazuya Okamoto, Wei Chen, and Xiang-Yang Li. Ranking of closeness centrality for large-scale social networks. In International Workshop on Frontiers in Algorithmics, pages 186-195. Springer, 2008.

[35] Joshua O'Madadhain and Padhraic Smyth. Eventrank: A framework for ranking time-varying networks. In Proceedings of the 3rd International Workshop on Link Discovery, pages 9-16. ACM, 2005.

[36] Joshua O'Madadhain, Jon Hutchins, and Padhraic Smyth. Prediction and ranking algorithms for event-based network data. ACM SIGKDD explorations Newsletter, 7(2):23-30, 2005.

[37] Xiaoyan Qiu, Diego FM Oliveira, Alireza Sahami Shirazi, Alessandro Flammini, and Filippo Menczer. Limited individual attention and online virality of low-quality information. Nature Human Behaviour, 1(7):0132, 2017.

[38] Filippo Radicchi and Claudio Castellano. Leveraging percolation theory to single out influential spreaders in networks. Physical Review E, 93(6):062314, 2016.

[39] Zhuo-Ming Ren, Manuel Sebastian Mariani, Yi-Cheng Zhang, and Matúš Medo. Randomizing growing networks with a time-respecting null model. Physical Review E, 97(5):052311, 2018.

[40] Bernard Roy. The outranking approach and the foundations of electre methods. In Readings in multiple criteria decision aid, pages 155-183. Springer, 1990.

[41] Thomas L Saaty. Analytic hierarchy process. In Encyclopedia of operations research and management science, pages 52-64. Springer, 2013.

[42] Matthew J Salganik and Duncan J Watts. Web-based experiments for the study of collective social dynamics in cultural markets. Topics in Cognitive Science, 1(3):439-468, 2009.

[43] Matthew J Salganik, Peter Sheridan Dodds, and Duncan J Watts. Experimental study of inequality and unpredictability in an artificial cultural market. Science, 311(5762):854-856, 2006. 
[44] David Schoch and Ulrik Brandes. Re-conceptualizing centrality in social networks. European Journal of Applied Mathematics, 27(6):971-985, 2016.

[45] Ingo Scholtes, René Pfitzner, and Frank Schweitzer. The social dimension of information ranking: A discussion of research challenges and approaches. In Socioinformatics-The Social Impact of Interactions between Humans and IT, pages 45-61. Springer, 2014.

[46] Ingo Scholtes, Nicolas Wider, René Pfitzner, Antonios Garas, Claudio J Tessone, and Frank Schweitzer. Causality-driven slow-down and speed-up of diffusion in non-markovian temporal networks. Nature Communications, 5:5024, 2014 .

[47] Ingo Scholtes, Nicolas Wider, and Antonios Garas. Higher-order aggregate networks in the analysis of temporal networks: path structures and centralities. The European Physical Journal B, 89(3):61, 2016.

[48] Amit Sharma, Jake M Hofman, and Duncan J Watts. Estimating the causal impact of recommendation systems from observational data. In Proceedings of the Sixteenth ACM Conference on Economics and Computation, pages 453470. ACM, 2015.

[49] Hirokazu Shirado and Nicholas A Christakis. Locally noisy autonomous agents improve global human coordination in network experiments. Nature, 545 (7654):370, 2017.

[50] Roberta Sinatra, Dashun Wang, Pierre Deville, Chaoming Song, and AlbertLászló Barabási. Quantifying the evolution of individual scientific impact. Science, 354(6312):aaf5239, 2016.

[51] Michele Tizzoni, Paolo Bajardi, Chiara Poletto, José J Ramasco, Duygu Balcan, Bruno Gonçalves, Nicola Perra, Vittoria Colizza, and Alessandro Vespignani. Real-time numerical forecast of global epidemic spreading: case study of 2009 a/h1n1pdm. BMC medicine, 10(1):165, 2012.

[52] Evangelos Triantaphyllou. Multi-criteria decision making methods. In Multicriteria decision making methods: A comparative study, pages 5-21. Springer, 2000.

[53] Giacomo Vaccario, Matúš Medo, Nicolas Wider, and Manuel Sebastian Mariani. Quantifying and suppressing ranking bias in a large citation network. Journal of Informetrics, 11(3):766-782, 2017.

[54] Ludo Waltman. A review of the literature on citation impact indicators. Journal of Informetrics, 10(2):365-391, 2016. 
[55] Dashun Wang, Chaoming Song, and Albert-László Barabási. Quantifying longterm scientific impact. Science, 342(6154):127-132, 2013.

[56] Lillian Weng, Alessandro Flammini, Alessandro Vespignani, and Fillipo Menczer. Competition among memes in a world with limited attention. Scientific Reports, 2:335, 2012.

[57] Jian Xu, Thanuka L Wickramarathne, and Nitesh V Chawla. Representing higher-order dependencies in networks. Science advances, 2(5):e1600028, 2016.

[58] Burcu Yucesoy, Xindi Wang, Junming Huang, and Albert-László Barabási. Success in books: a big data approach to bestsellers. EPJ Data Science, 7(1): 7, 2018.

[59] An Zeng, Chi Ho Yeung, Matúš Medo, and Yi-Cheng Zhang. Modeling mutual feedback between users and recommender systems. Journal of Statistical Mechanics: Theory and Experiment, 2015(7):P07020, 2015.

[60] Zhihui Zhang, Ying Cheng, and Nian Cai Liu. Comparison of the effect of mean-based method and z-score for field normalization of citations at the level of web of science subject categories. Scientometrics, 101(3):1679-1693, 2014.

[61] Tao Zhou, Zoltán Kuscsik, Jian-Guo Liu, Matúš Medo, Joseph Rushton Wakeling, and Yi-Cheng Zhang. Solving the apparent diversity-accuracy dilemma of recommender systems. Proceedings of the National Academy of Sciences, 107(10):4511-4515, 2010.

\section{Appendix A. Details on the numerical simulations}

We focus on networks composed of $10^{4}$ nodes, and study the following model parameters: $\alpha$ from 0.25 to 2.0 with step 0.25 and $\beta$ from 0 to 1 with step 0.1 . Node quality values are drawn from the Pareto distribution $P(q) \sim q^{-3}$ where $q \in[1, \infty)$. The network is initialized with a network of $m$ nodes, each of them with one outgoing and incoming link. At each time step $t>m+1$, a new node $t$ is added to the system and $m$ nodes (only results for $m=6$ are shown in main text, whereas results for both $m=3$ and $m=6$ are shown in the Supplementary Material) are chosen as targets to establish $m$ new links. With probability $\beta$, the probability that a given node is chosen is given by Eq. (1), with probability $1-\beta$, it is given by Eq. (2). To save computational time, for times $t \leq 10^{2}$, we re-compute and update the rankings at each time step, whereas for times $t \geq 10^{2}$, the newly introduced nodes are placed at the bottom of the node ranking, and we re-compute and update the rankings every 10 time steps. To make our results insensitive to 
random fluctuations, for each parameter pair $(\alpha, \beta)$, all the results shown here represent averages over a sufficiently large number of realizations of the network growth process.

\section{Appendix B. The relation between popularity and quality in indegree- generated networks}

We start by considering networks where the nodes cannot perceive the other nodes ranking, and are completely driven by quality $(\beta=1)$. In this scenario, the average indegree of node $i$ at time $N$ is given by

$$
\overline{k_{i}}(N)=\sum_{t=i+1}^{N} m \frac{q_{i}}{\sum_{j=1}^{t-1} q_{j}} .
$$

In the thermodynamic limit $N \gg i$, by using a similar mean-field approximation as in [12], we obtain

$$
\overline{k_{i}}(N) \simeq m \frac{q_{i}}{\bar{q}} \log \left(\frac{N-1}{i-1}\right)
$$

There is a good agreement between Eq. (B.2) and the results of numerical simulations (see Supplementary Fig. S26). Such linear relation between indegree and quality does not hold for $\beta>0$, where the analytic calculation is made difficult by the fact that the ranking position of a given node at a given time is influenced by both its quality and the previous dynamics of the system. Nevertheless, we find that the relation $\overline{k_{i}}(N)=C q_{i}^{\delta}$ fits reasonably well the simulation results, and the dependence of the fitted exponent $\delta$ on node age is relatively weak (see Fig. S27 and Table S1 for details). 


\section{Supplemental Materials: \\ The long-term impact of ranking algorithms in growing networks}
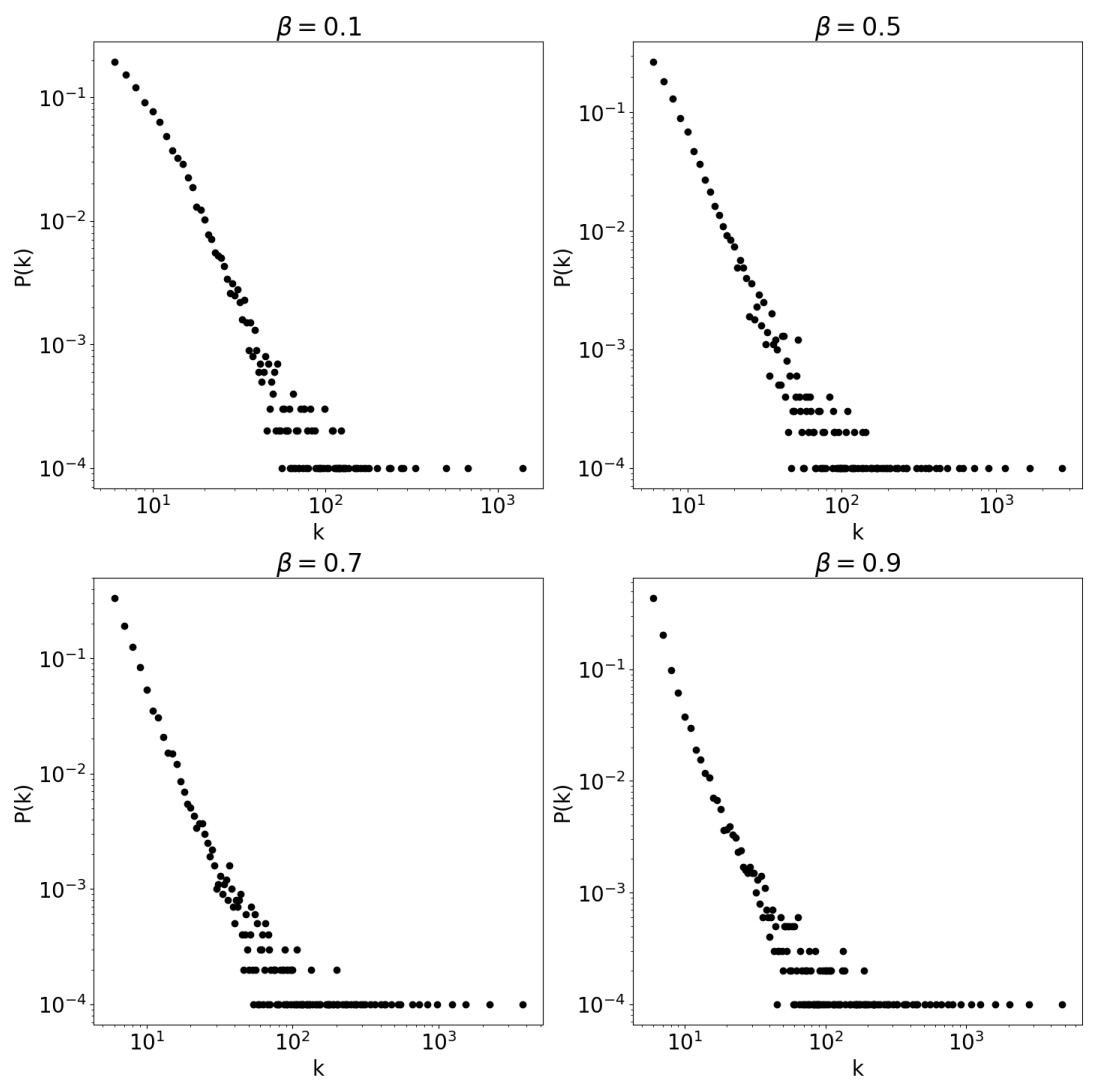

Figure S1: Results for $m=6$. The degree distribution of indegree-generated networks, the four panels correspond to for $\beta=0.1,0.5,0.7,0.9$ with fixed $\alpha=1.0$ respectively. 
A)
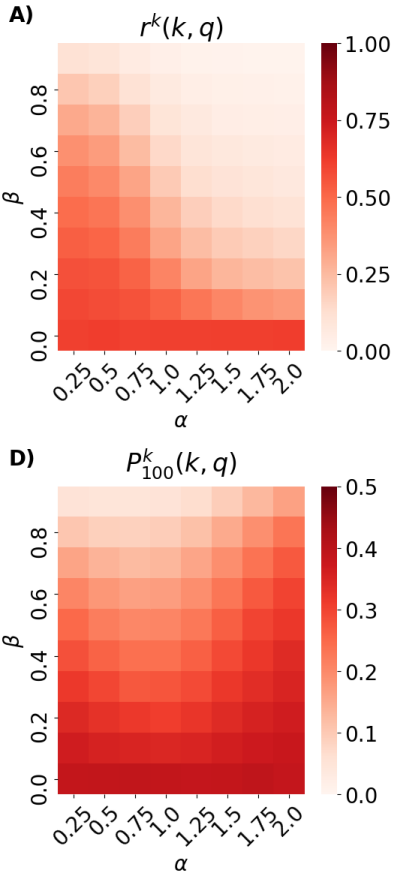

B)

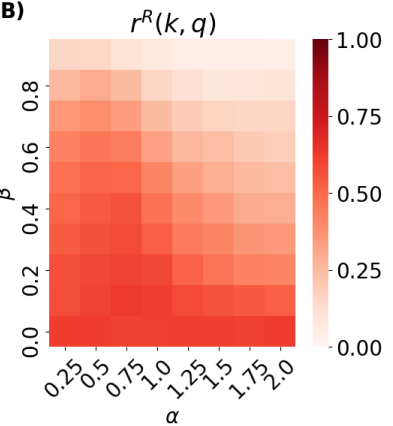

E)

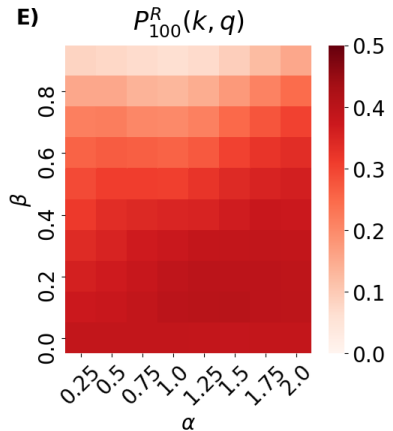

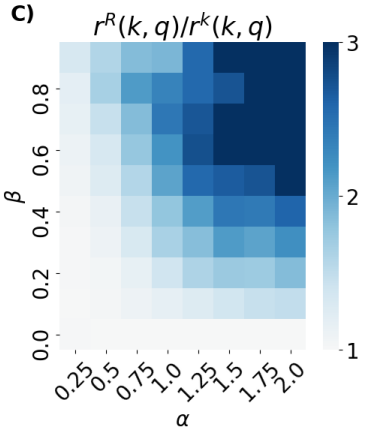

F) $\quad P_{100}^{R}(k, q) / P_{100}^{k}(k, q)$

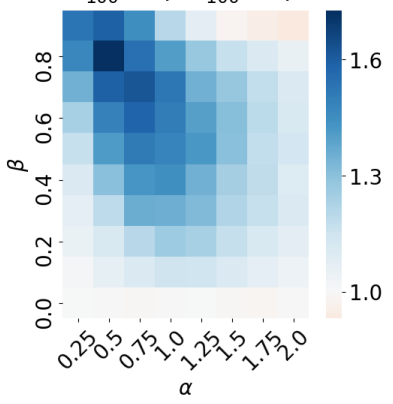

Figure S2: Results for $m=3$. Quality promotion as measured by $r(k, q)$ (the Pearson's linear correlation between node indegree $k$ and node quality $q$ ), and $P_{100}(k, q)$ (the precision of node indegree $k$ in identifying the top-100 nodes by quality $q$-bottom panels): comparison between indegree-generated and $R(k)$-generated networks. (A-B): $r(k, q)$ for indegree-generated $\left(r^{k}(k, q)\right.$, panel A) and $R(k)$-generated $\left(r^{R}(k, q)\right.$, panel B) networks, as a function of the model's parameter $\alpha$ (exploration cost) and $\beta$ (reliance on ranking). (C): Ratio $r^{R}(k, q) / r^{k}(k, q)$ as a function of the model's parameters. (D-E): $P_{100}(k, q)$ for indegree-generated $\left(P_{100}^{k}(k, q)\right.$, panel D) and $R(k)$ generated $\left(P_{100}^{R}(k, q)\right.$, panel E) networks, as a function of the model's parameters. (F): Ratio $P_{100}^{R}(k, q) / P_{100}^{k}(k, q)$ as a function of the model's parameters. Results are averaged over 500 realizations. 

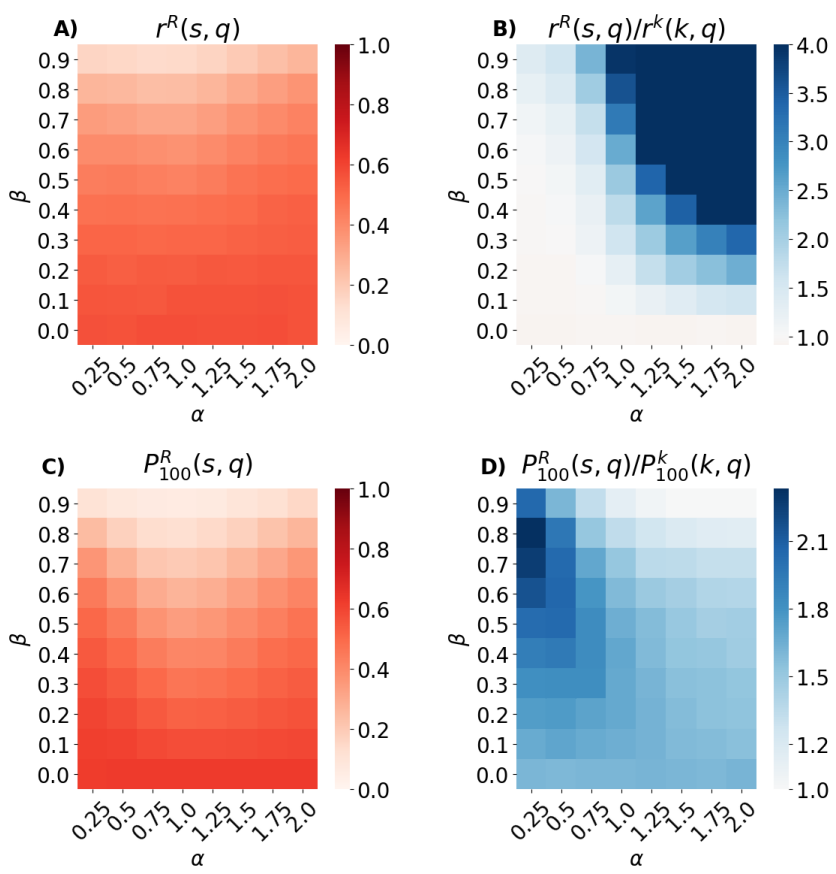

Figure S3: Results for $m=3$. Quality detection as measured by $r(s, q)$ (the Pearson's linear correlation between node score $s$ and node quality $q$-top panels), and $P_{100}(s, q)$ (the precision of node score $s$ in identifying the top-100 nodes by quality $q$-bottom panels): comparison between indegree-generated and $R(k)$-generated networks. (A) $r(s, q)$ for $R(k)$-generated networks, as a function of the model's parameter $\alpha$ (exploration cost) and $\beta$ (popularity bias). (B) Ratio $r^{R}(s, q) / r^{k}(k, q)$ as a function of the model's parameters. (C) $P_{100}(s, q)$ for $R(k)$-generated networks, as a function of the model's parameters. (D) Ratio $P_{100}^{R}(s, q) / P_{100}^{k}(k, q)$ as a function of the model's parameters. Results are averaged over 500 realizations.
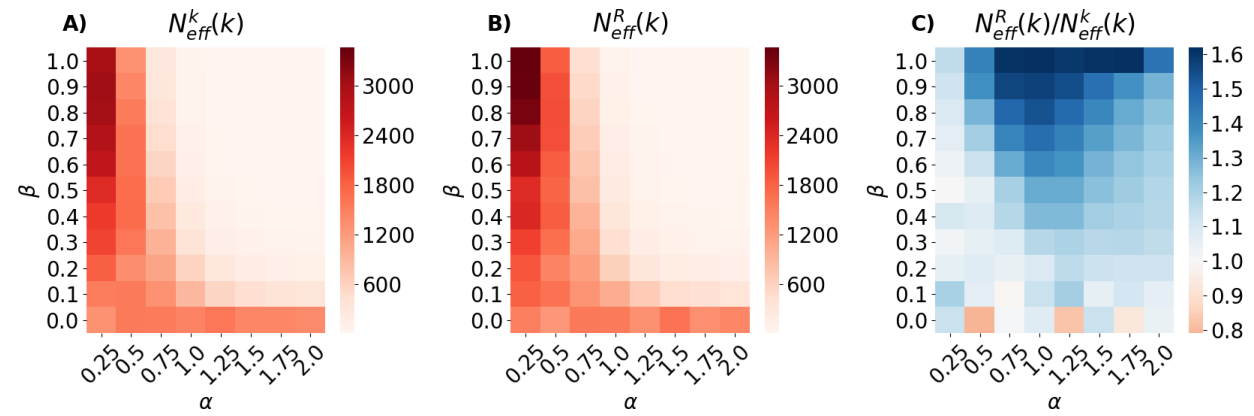

Figure S4: Results for $m=3$. Diversity as measured by $N_{\text {eff }}$ (the larger, the more egalitarian the indegree distribution): comparison between popularity-generated and $R(k)$-generated networks. (A-B) The effective number of nodes $N_{e f f}(k)$ for indegree-generated $\left(N_{e f f}^{k}(k)\right.$, panel A) and $R(k)$-generated ( $N_{e f f}^{R}(k)$, panel B) networks, as a function of the model's parameters. (C) Ratio $N_{e f f}^{R}(k) / N_{e f f}^{k}(k)$ as a function of the model's parameters. Results are averaged over 500 realizations. 

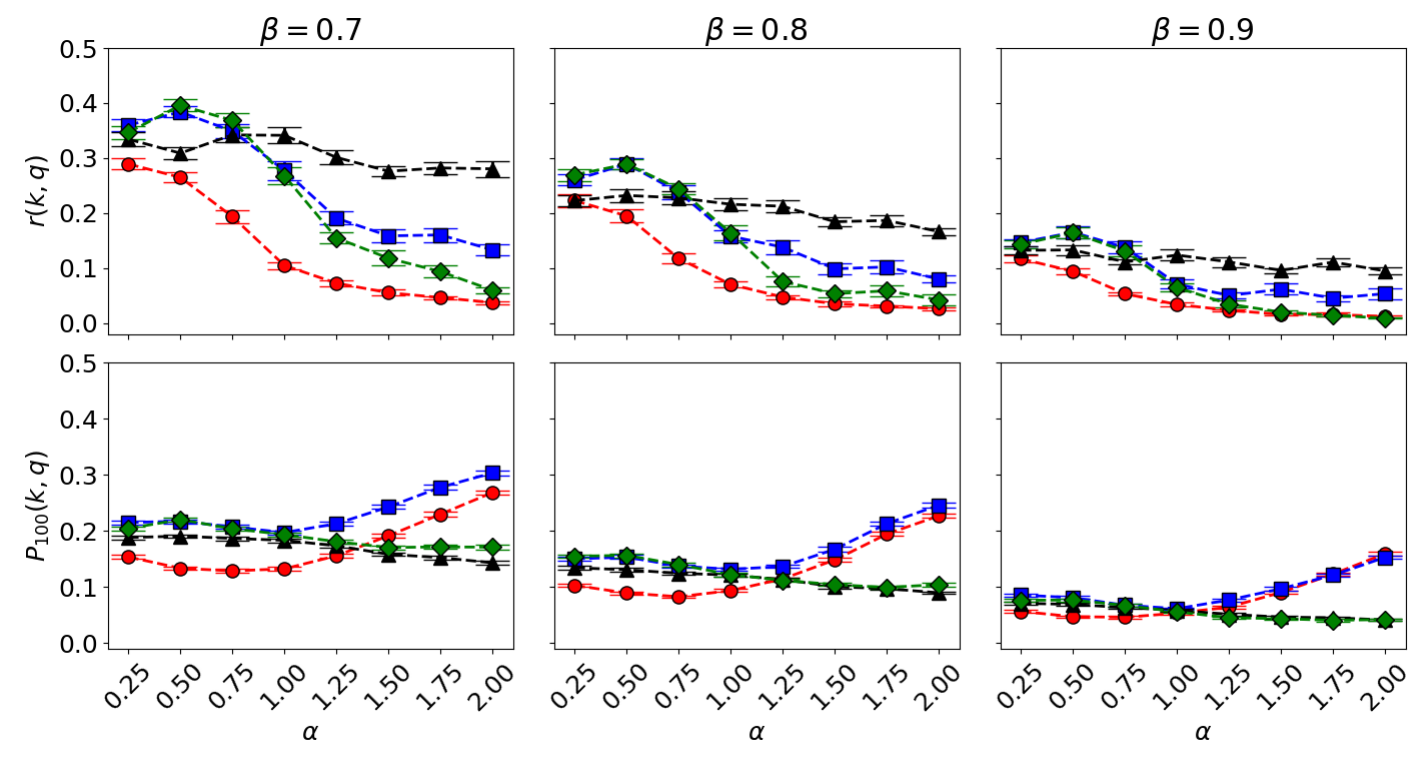

Figure S5: Results for $m=3$. Quality promotion as measured by $r(k, q)$ (the Pearson's linear correlation between node indegree $k$ and node quality $q$-top panels), and $P_{100}(k, q)$ (the precision of node indegree $k$ in identifying the top-100 nodes by quality $q$ - bottom panels): comparison between indegree-generated (circles), $R(k)$-generated (squares), $R(k)+R P$-generated (green rhombuses), and random-generated networks (triangles). The three columns correspond, from left to right, to $\beta=0.7,0.8,0.9$, respectively. Results are averaged over 100 realizations; the error bars represent the standard error of the mean. 

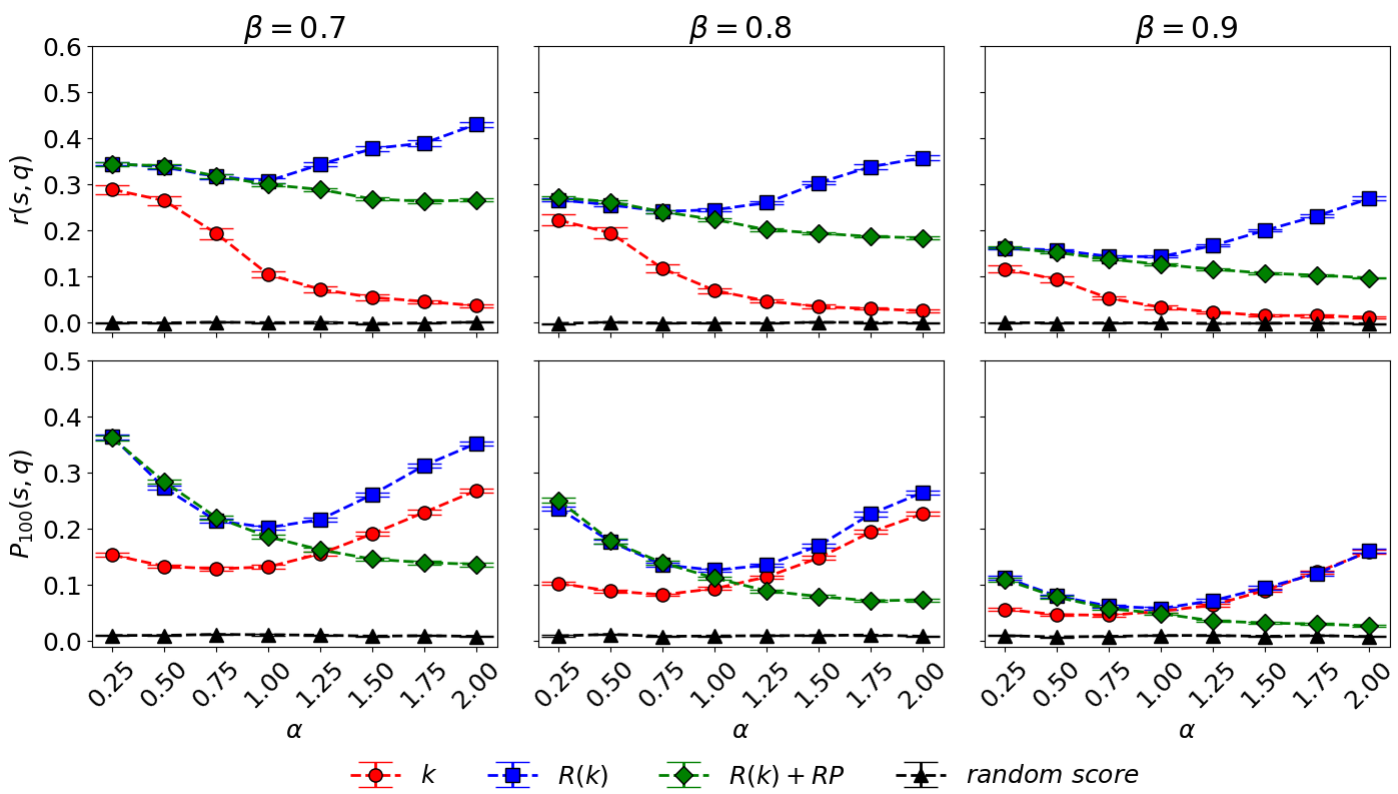

$R(k)$

- ㄷ. $R(k)+R P$

F- random score

Figure S6: Results for $m=3$. Quality detection as measured by $r(s, q)$ (the Pearson's linear correlation between node score $s$ and node quality $q$-top panels), and $P_{100}(s, q)$ (the precision of node score $s$ in identifying the top-100 nodes by quality $q$ - bottom panels): comparison between indegree-generated ( $s=k$, circles), $R(k)$-generated ( $s=R(k)$, squares), $R(k)+R P$ generated (green rhombuses), and random-generated networks ( $s=\rho$, triangles). The three columns correspond, from left to right, to $\beta=0.7,0.8,0.9$, respectively. The dots represent averages over 100 realizations; the error bars represent the standard error of the mean.
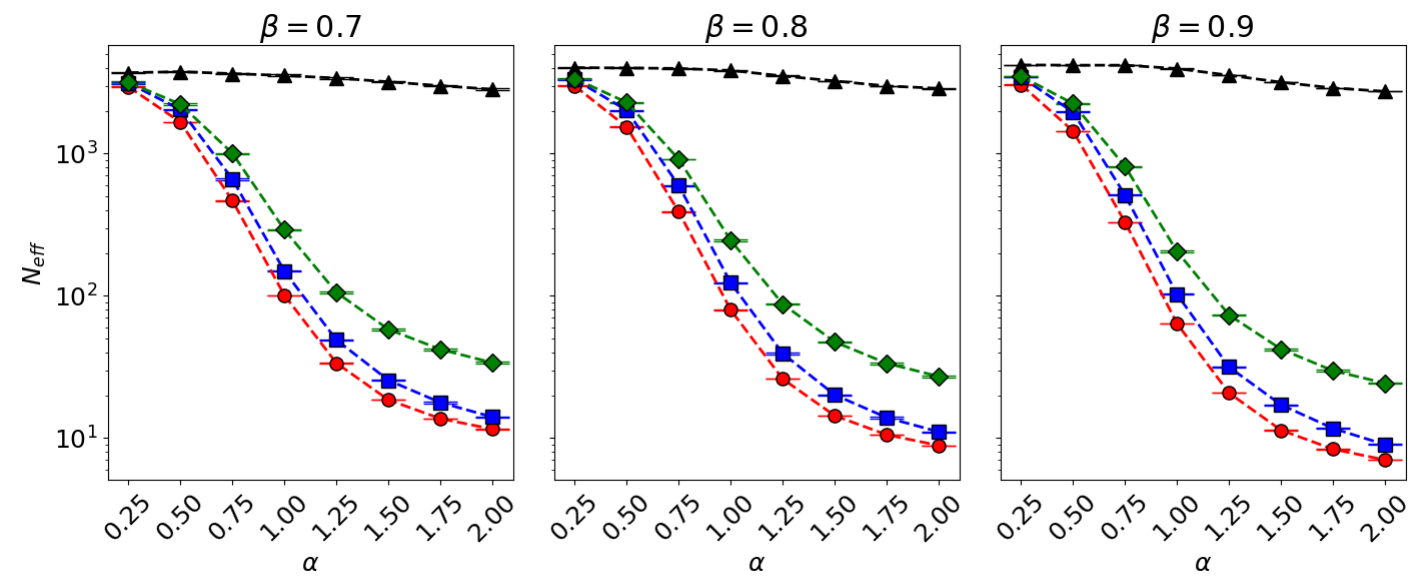

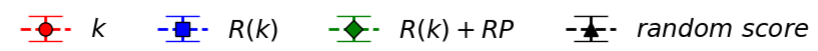

Figure S7: Results for $m=3$. Diversity as measured by $N_{\text {eff }}$ (the larger, the more egalitarian the indegree distribution): comparison between indegree-generated (circles), $R(k)$-generated (squares), $R(k)+R P$-generated (green rhombuses), and random-generated (triangles) networks. The three columns correspond, from left to right, to $\beta=0.7,0.8,0.9$, respectively. The dots represent averages over 100 realizations; the error bars represent the standard error of the mean. 

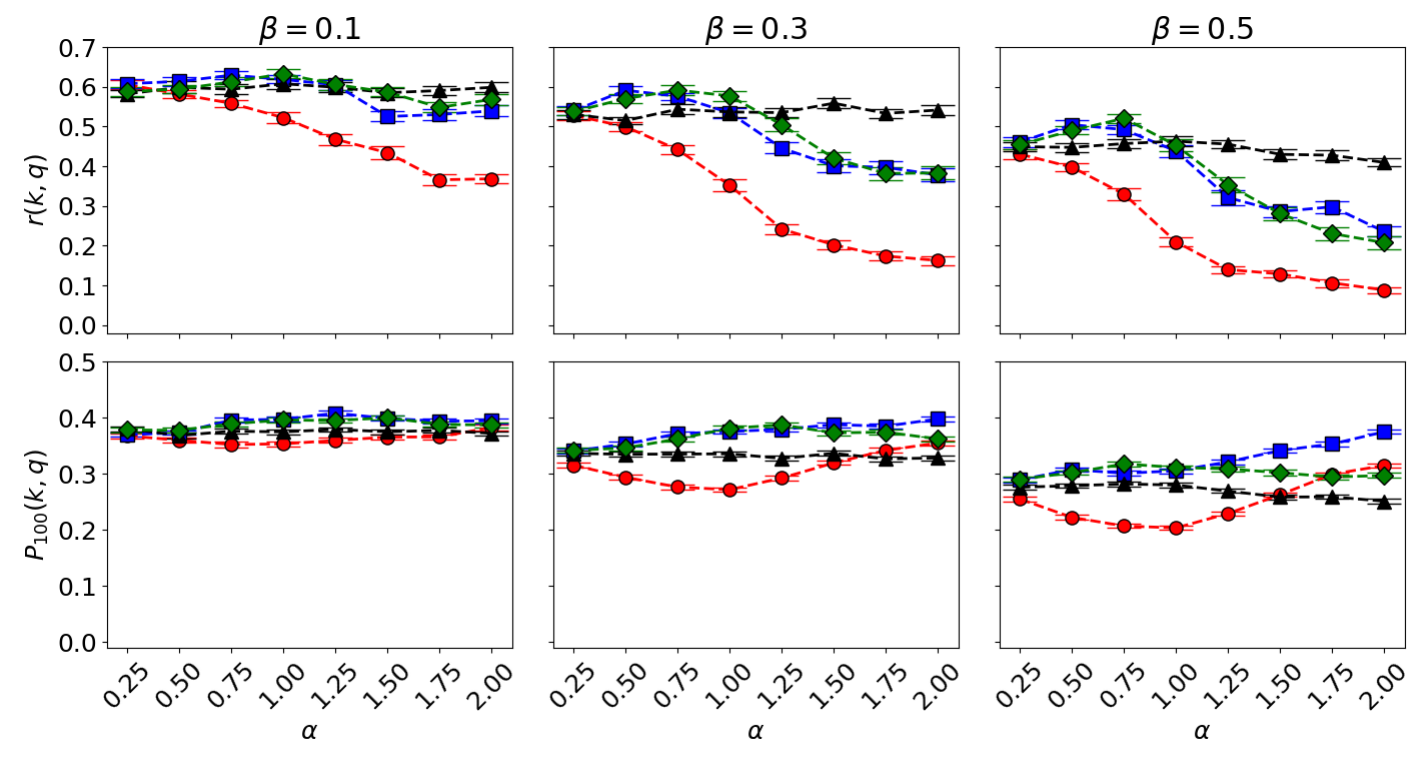

Figure S8: Results for $m=3$. Quality promotion as measured by $r(k, q)$ (the Pearson's linear correlation between node indegree $k$ and node quality $q$-top panels), and $P_{100}(k, q)$ (the precision of node indegree $k$ in identifying the top-100 nodes by quality $q$ - bottom panels): comparison between indegree-generated (circles), $R(k)$-generated (squares), $R(k)+R P$-generated (green rhombuses), and random-generated networks (triangles). The three columns correspond, from left to right, to $\beta=0.1,0.3,0.5$, respectively. Results are averaged over 100 realizations; the error bars represent the standard error of the mean. 

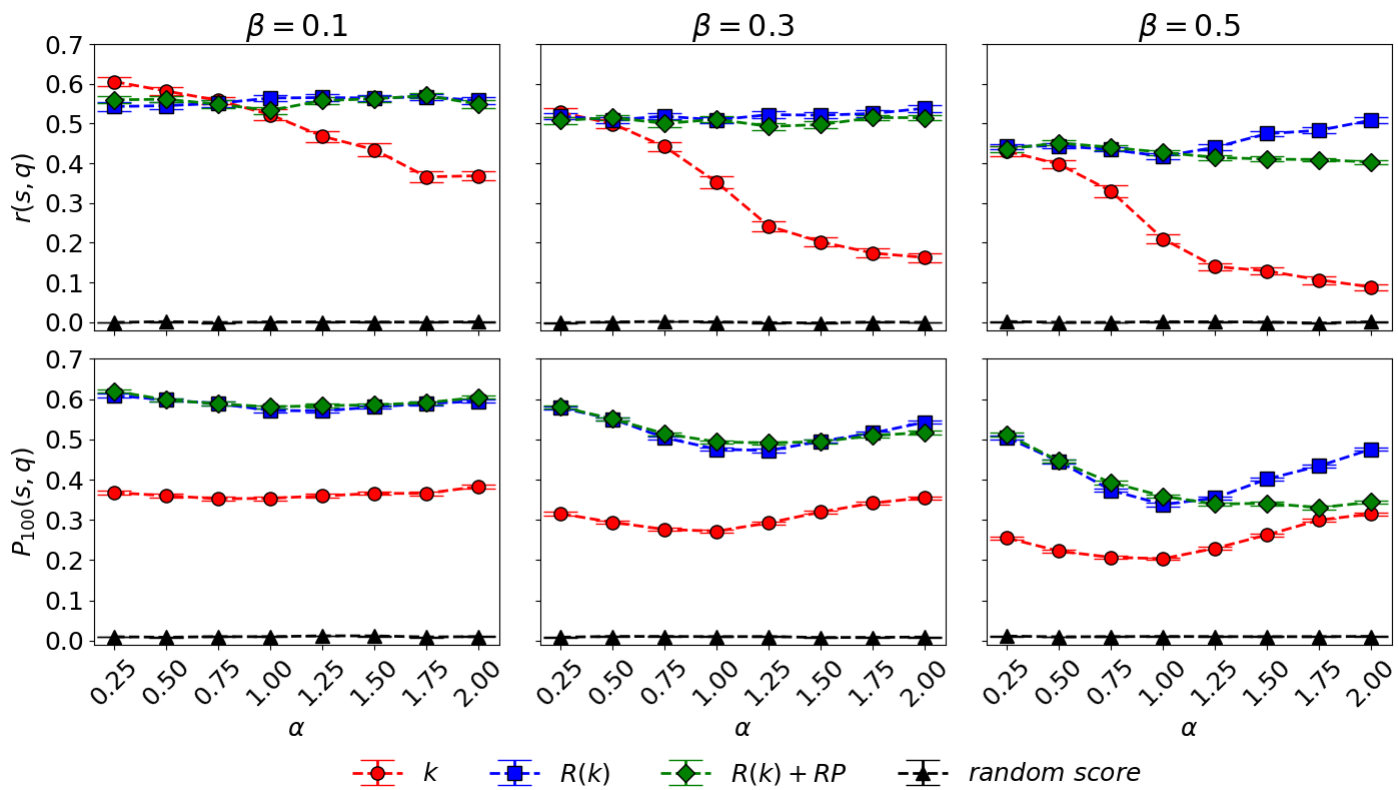

Figure S9: Results for $m=3$. Quality detection as measured by $r(s, q)$ (the Pearson's linear correlation between node score $s$ and node quality $q$-top panels), and $P_{100}(s, q)$ (the precision of node score $s$ in identifying the top-100 nodes by quality $q$-bottom panels): comparison between indegree-generated ( $s=k$, circles), $R(k)$-generated $(s=R(k), R(k)+R P$-generated (green rhombuses), squares), and random-generated networks ( $s=\rho$, triangles). The three columns correspond, from left to right, to $\beta=0.1,0.3,0.5$, respectively. The dots represent averages over 100 realizations; the error bars represent the standard error of the mean.
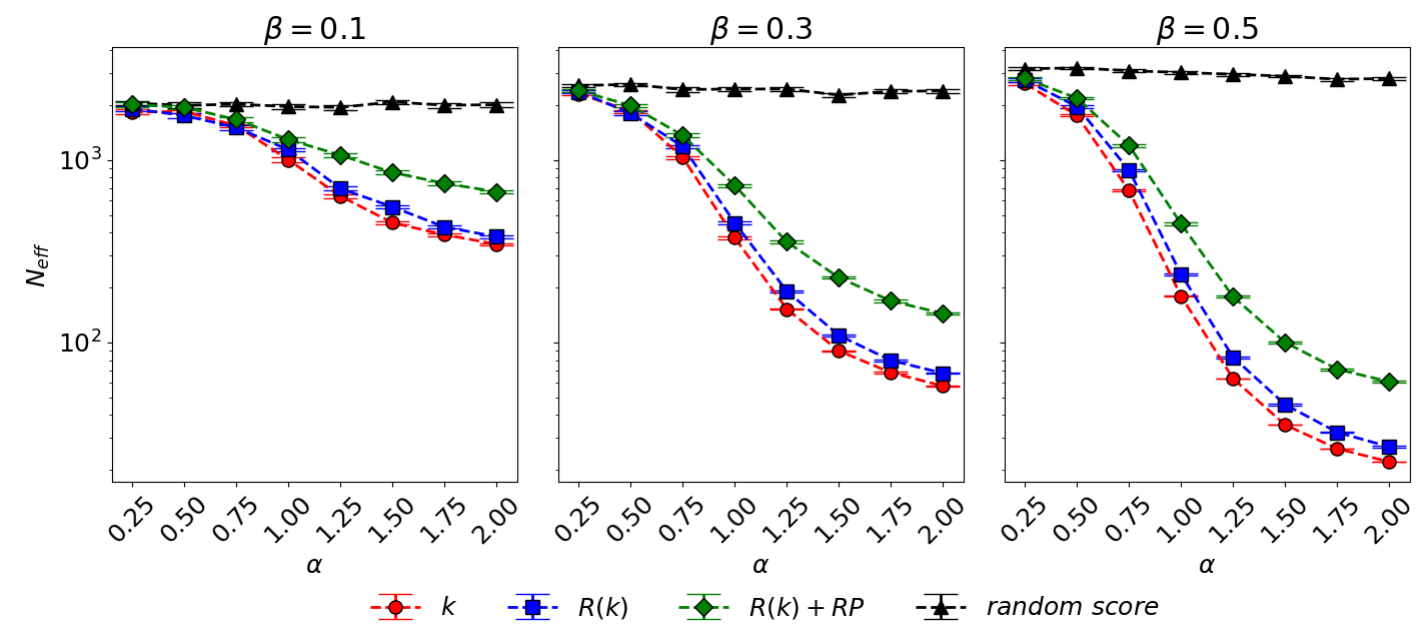

Figure S10: Results for $m=3$. Diversity as measured by the effective number of nodes $N_{\text {eff }}$ (the larger, the more egalitarian the indegree distribution): comparison between indegreegenerated (circles), $R(k)$-generated (squares), $R(k)+R P$-generated (green rhombuses), and random-generated (triangles) networks. The three columns correspond, from left to right, to $\beta=0.1,0.3,0.5$, respectively. The dots represent averages over 100 realizations; the error bars represent the standard error of the mean. 

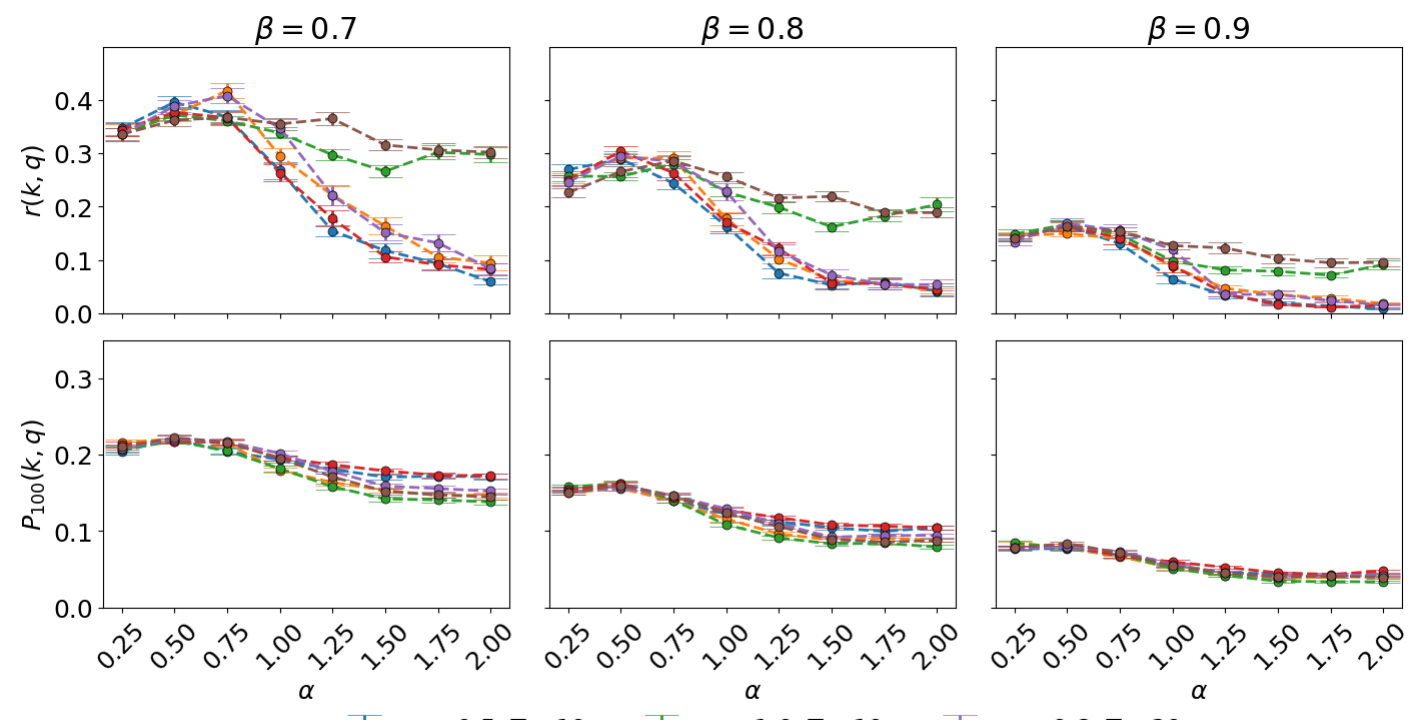

-क- $\quad \eta=0.5, T=10 \quad \eta \quad \phi=1.0, T=10 \quad-\phi=0.8, T=20$

$\eta+\phi=0.8, T=10 \quad \eta=0.5, T=20 \quad-\phi=1.0, T=20$

Figure S11: Results for $m=3$. Quality promotion as measured by $r(k, q)$ (the Pearson's linear correlation between node indegree $k$ and node quality $q$-top panels), and $P_{100}(k, q)$ (the precision of node indegree $k$ in identifying the top-100 nodes by quality $q$-bottom panels): comparison between $R(k)+R P$-generated networks with different parameters $\eta=P / T=$ $5 / 10,8 / 10,10 / 10,10 / 20,16 / 20,20 / 20$. The three columns correspond, from left to right, to $\beta=0.7,0.8,0.9$, respectively. The dots represent averages over 100 realizations; the error bars represent the standard error of the mean. 

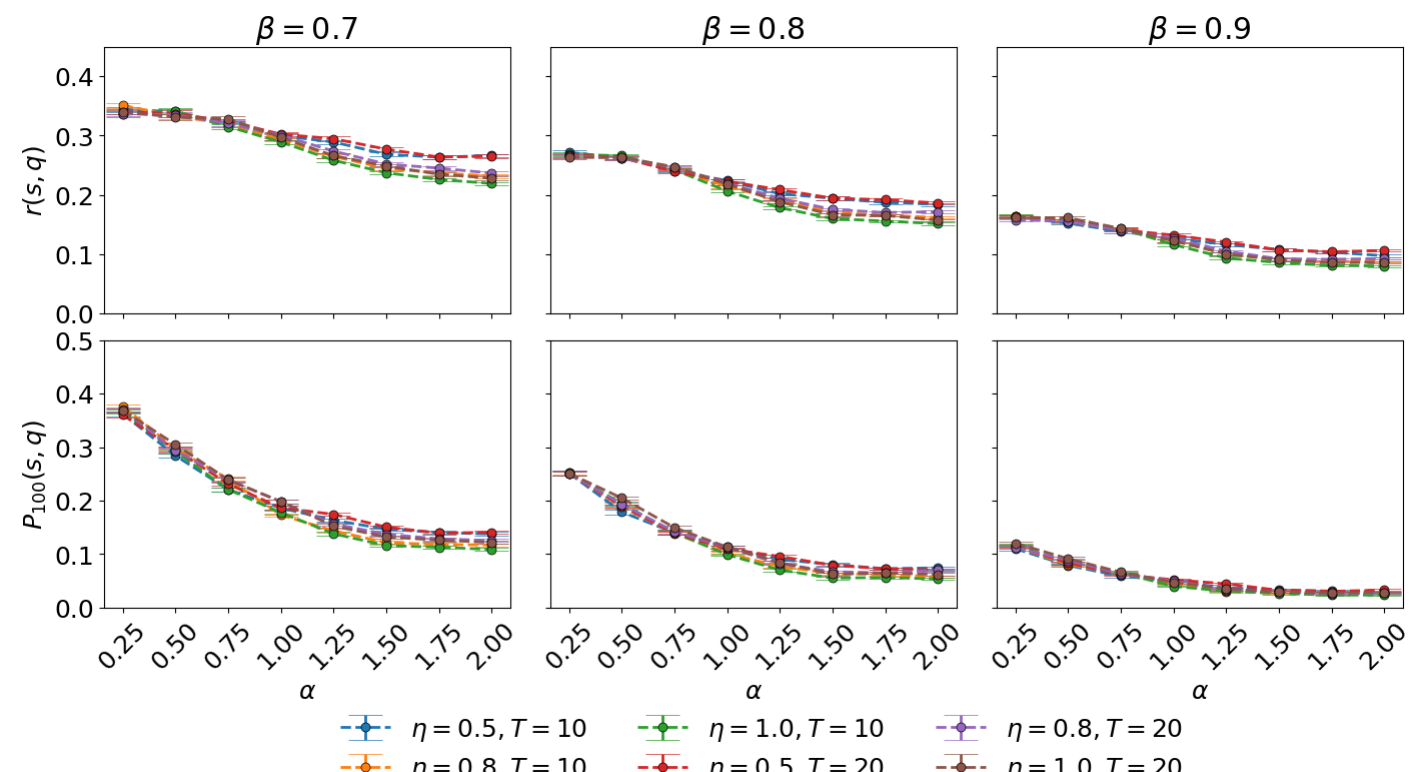

$$
\eta=0.8, T=10 \quad \eta=0.5, T=20 \quad \eta=1.0, T=20
$$

Figure S12: Results for $m=3$. Quality detection as measured by $r(s, q)$ (the Pearson's linear correlation between node score $s$ and node quality $q$-top panels), and $P_{100}(s, q)$ (the precision of node score $s$ in identifying the top-100 nodes by quality $q$ - bottom panels): comparison between $R(k)+R P$-generated networks with different parameters $\eta=P / T=$ $5 / 10,8 / 10,10 / 10,10 / 20,16 / 20,20 / 20$. The three columns correspond, from left to right, to $\beta=0.7,0.8,0.9$, respectively. The dots represent averages over 100 realizations; the error bars represent the standard error of the mean. 

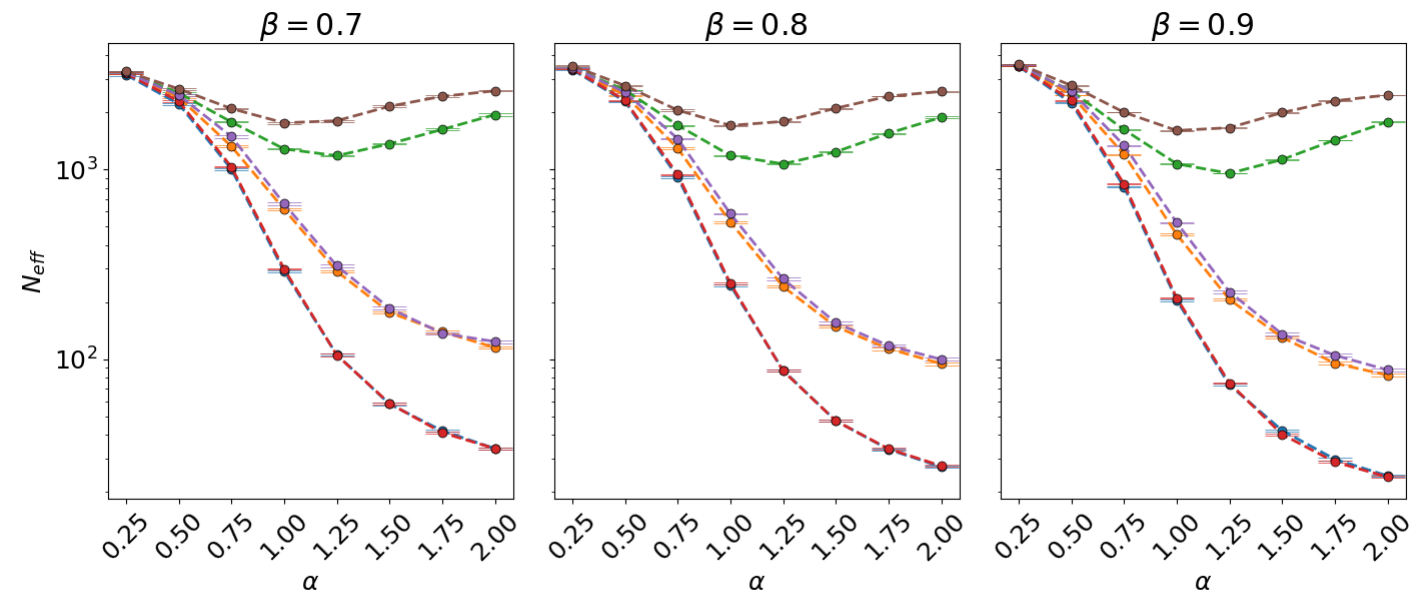

$$
\begin{aligned}
& \eta=0.5, T=10 \quad \eta=1.0, T=10 \quad \eta \quad-\phi=0.8, T=20 \\
& \eta=0.8, T=10 \quad \eta=0.5, T=20 \quad \eta=1.0, T=20
\end{aligned}
$$

Figure S13: Results for $m=3$. Diversity as measured by the effective number of nodes $N_{\text {eff }}$ (the larger, the more egalitarian the indegree distribution): comparison between $R(k)+R P$-generated networks with different parameters $\eta=P / T=5 / 10,8 / 10,10 / 10,10 / 20,16 / 20,20 / 20$. The three columns correspond, from left to right, to $\beta=0.7,0.8,0.9$, respectively. The dots represent averages over 100 realizations; the error bars represent the standard error of the mean. 

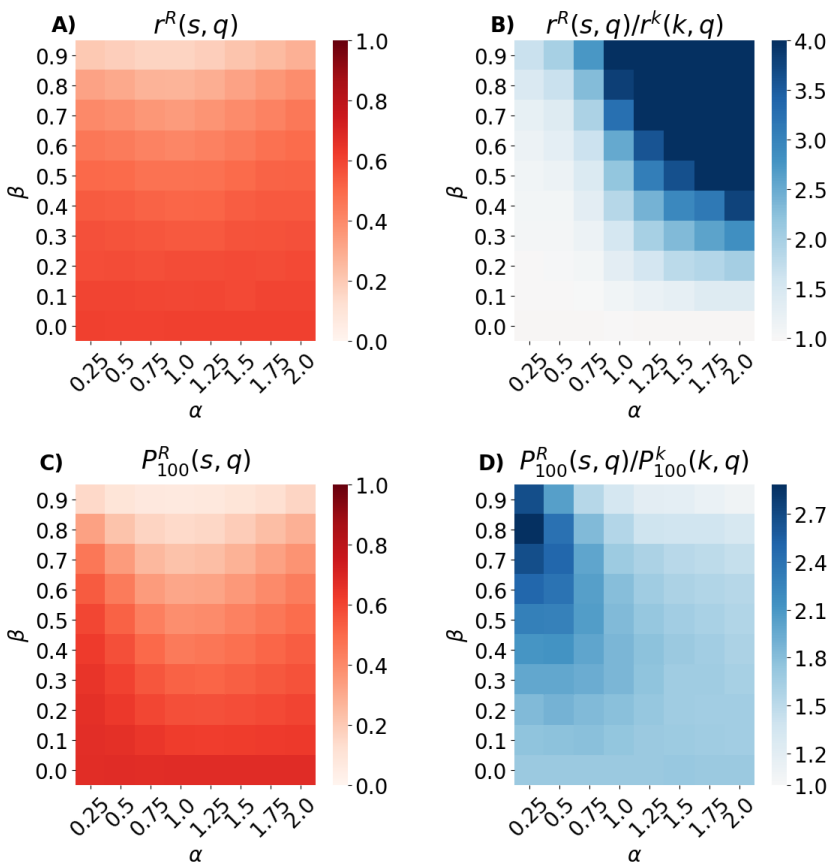

Figure S14: Results for $m=6$. Quality detection as measured by $r(s, q)$ (the Pearson's linear correlation between node score $s$ and node quality $q$-top panels), and $P_{100}(s, q)$ (the precision of node score $s$ in identifying the top-100 nodes by quality $q$ - bottom panels): comparison between popularity-generated and $R(k)$-generated networks. (A) $r^{R}(s, q)$ for $R(k)$-generated networks, as a function of the model parameters $\alpha$ (exploration cost) and $\beta$ (popularity bias). (B) Ratio $r^{R}(s, q) / r^{k}(k, q)$ as a function of the model parameters. (C) $P_{100}(s, q)$ for $R(k)$-generated networks, as a function of the model parameters. (D) Ratio $P_{100}^{R}(s, q) / P_{100}^{k}(k, q)$ as a function of the model parameters. Results are averaged over 500 realizations.
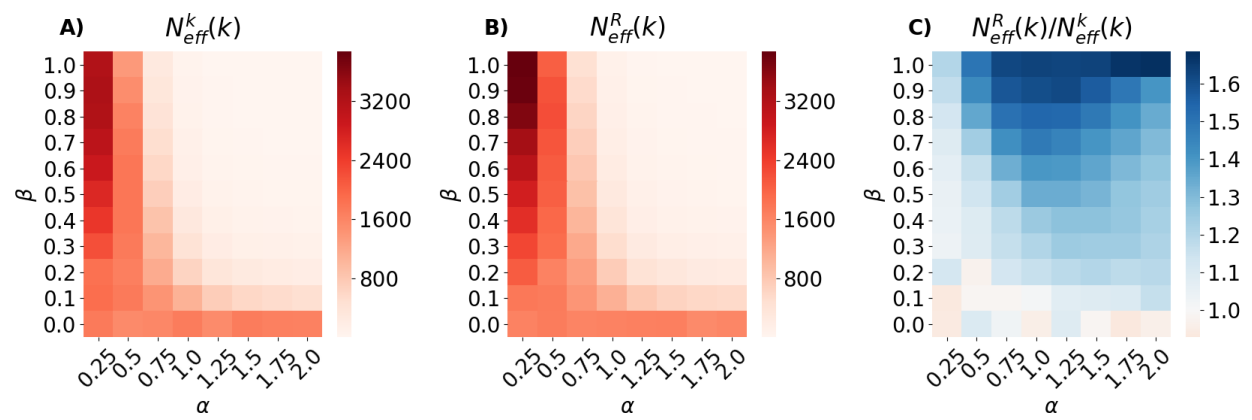

Figure S15: Results for $m=6$. Diversity as measured by the effective number of nodes: comparison between popularity-generated and $R(k)$-generated networks. (A-B) The effective number of nodes $N_{e f f}(k)$ for indegree-generated $\left(N_{e f f}^{k}(k)\right.$, panel A) and $R(k)$-generated $\left(N_{e f f}^{R}(k)\right.$, panel B) networks, as a function of the model parameters. (C) Ratio $N_{e f f}^{R}(k) / N_{e f f}^{k}(k)$ as a function of the model parameters. Results are averaged over 500 realizations. 



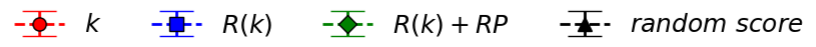

Figure S16: Results for $m=6$. Quality promotion as measured by $r(k, q)$ (the Pearson's linear correlation between node indegree $k$ and node quality $q$-top panels), and $P_{100}(k, q)$ (the precision of node indegree $k$ in identifying the top-100 nodes by quality $q$-bottom panels): comparison between indegree-generated (circles), $R(k)$-generated (squares), $R(k)+R P$-generated (green rhombuses), and random-generated networks (triangles). The three columns correspond, from left to right, to $\beta=0.1,0.3,0.5$, respectively. Results are averaged over 100 realizations; the error bars represent the standard error of the mean. 

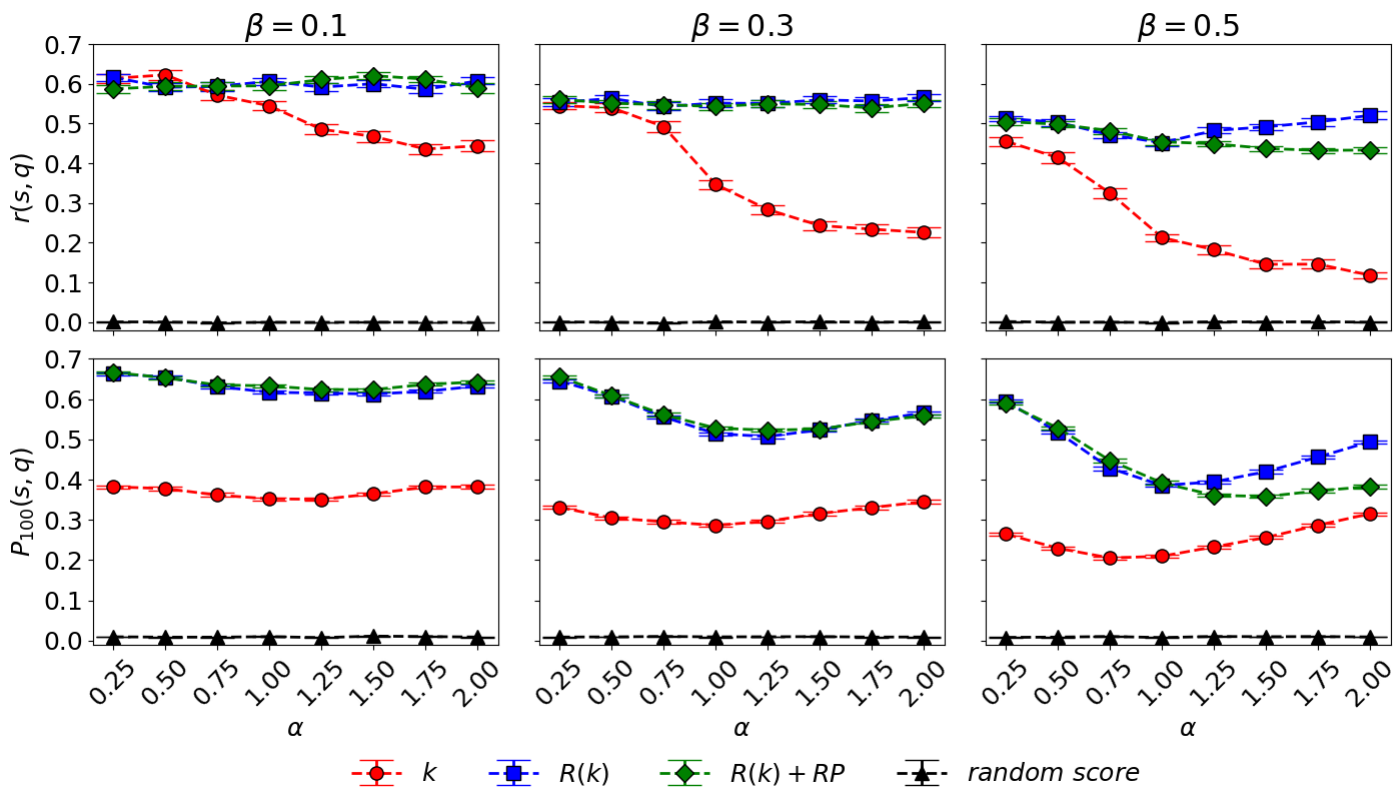

$R(k)$

-다. $R(k)+R P$-다. random score

Figure S17: Results for $m=6$. Quality detection as measured by $r(s, q)$ (the Pearson's linear correlation between node score $s$ and node quality $q$-top panels), and $P_{100}(s, q)$ (the precision of node score $s$ in identifying the top-100 nodes by quality $q$-bottom panels): comparison between indegree-generated ( $s=k$, circles), $R(k)$-generated $(s=R(k)$, squares), $R(k)+R P$ generated (green rhombuses), and random-generated networks ( $s=\rho$, triangles). The three columns correspond, from left to right, to $\beta=0.1,0.3,0.5$, respectively. The dots represent averages over 100 realizations; the error bars represent the standard error of the mean.
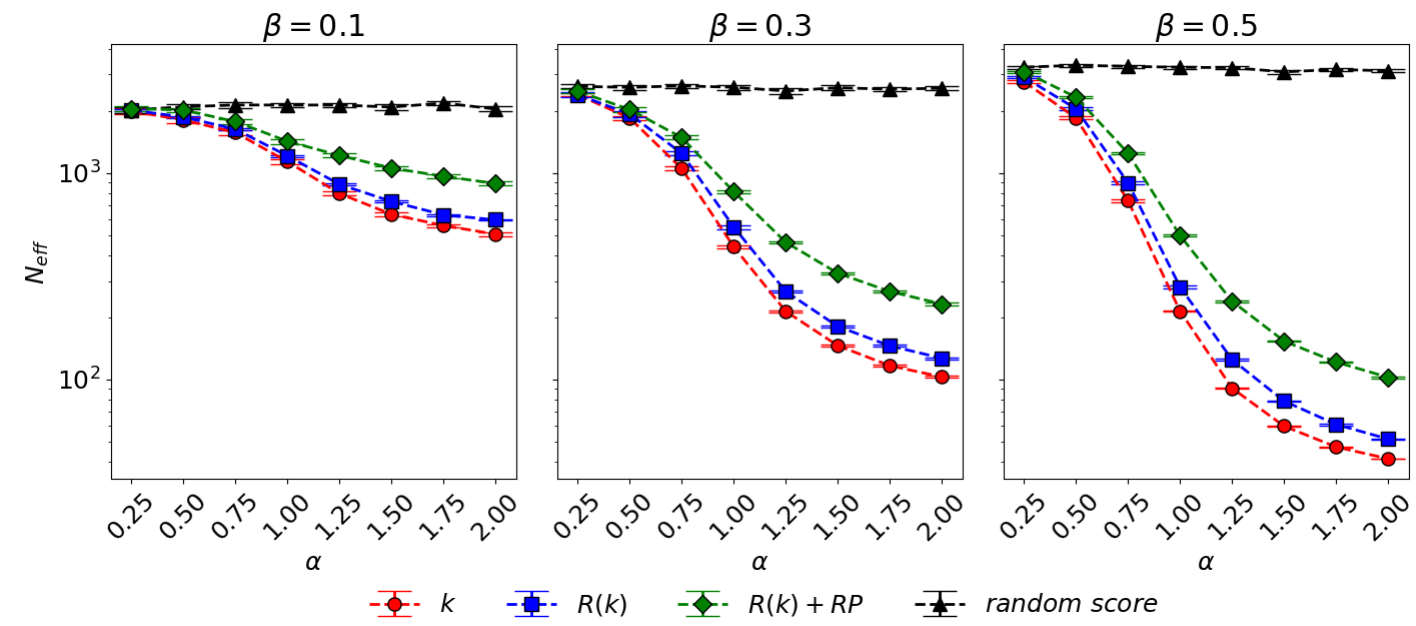

Figure S18: Results for $m=6$. Diversity as measured by the effective number of nodes $N_{\text {eff }}$ (the larger, the more egalitarian the indegree distribution): comparison between indegreegenerated (circles), $R(k)$-generated (squares), $R(k)+R P$-generated (green rhombuses), and random-generated (triangles) networks. The three columns correspond, from left to right, to $\beta=0.1,0.3,0.5$, respectively. The dots represent averages over 100 realizations; the error bars represent the standard error of the mean. 



Figure S19: Results for $m=6$. Quality promotion as measured by $r(k, q)$ (the Pearson's linear correlation between node indegree $k$ and node quality $q$-top panels), and $P_{100}(k, q)$ (the precision of node indegree $k$ in identifying the top-100 nodes by quality $q$ - bottom panels): comparison between $R(k)+R P$-generated networks with different parameters $\eta=P / T=$ $5 / 10,8 / 10,10 / 10,10 / 20,16 / 20,20 / 20$. The three columns correspond, from left to right, to $\beta=0.1,0.3,0.5$, respectively. The dots represent averages over 100 realizations; the error bars represent the standard error of the mean. 

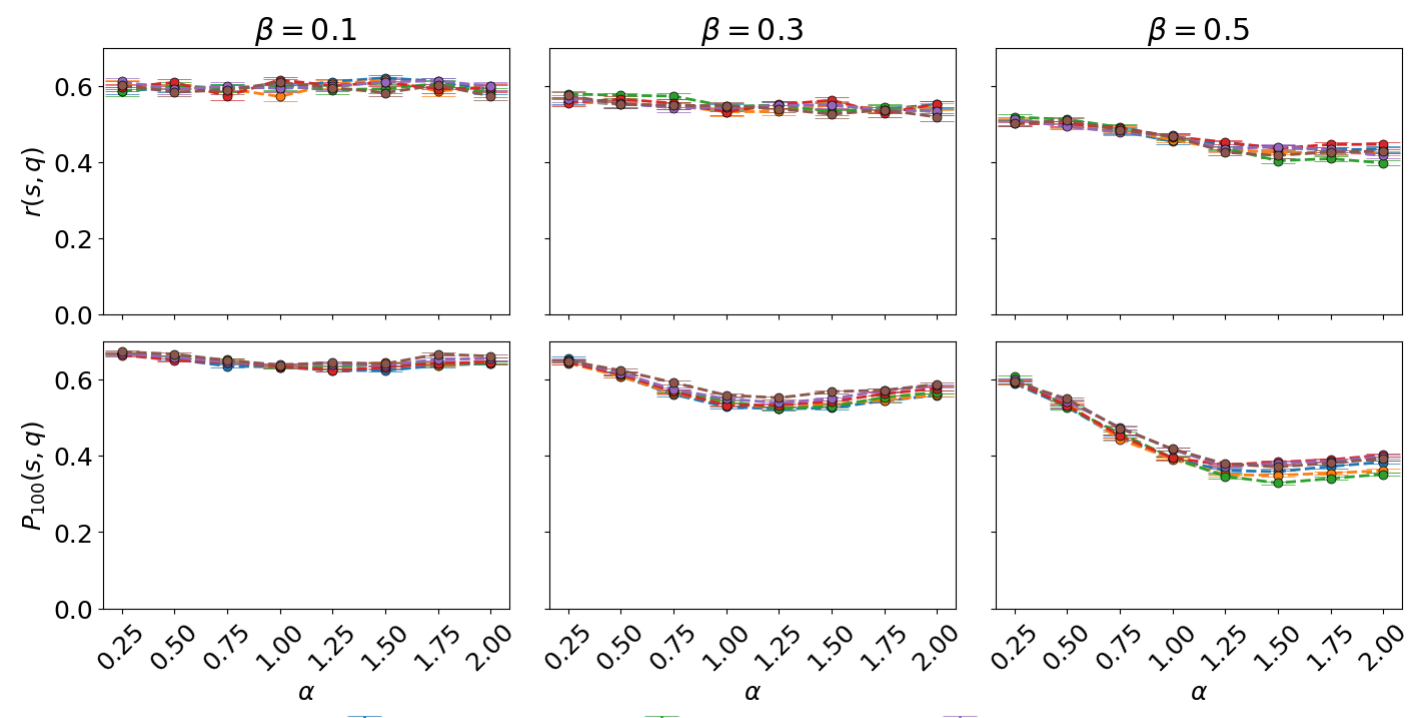



Figure S20: Results for $m=6$. Quality detection as measured by $r(s, q)$ (the Pearson's linear correlation between node score $s$ and node quality $q$-top panels), and $P_{100}(s, q)$ (the precision of node score $s$ in identifying the top-100 nodes by quality $q$ - bottom panels): comparison between $R(k)+R P$-generated networks with different parameters $\eta=P / T=$ $5 / 10,8 / 10,10 / 10,10 / 20,16 / 20,20 / 20$. The three columns correspond, from left to right, to $\beta=0.1,0.3,0.5$, respectively. The dots represent averages over 100 realizations; the error bars represent the standard error of the mean. 

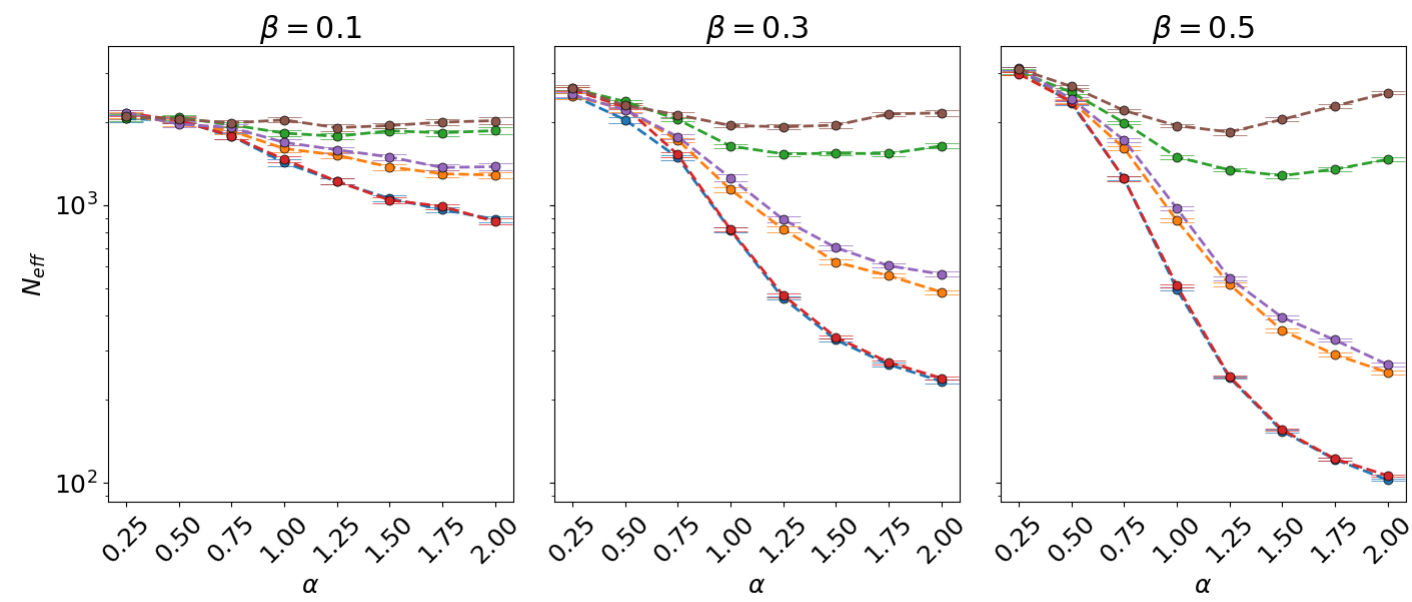

$$
\begin{aligned}
& \eta=0.5, T=10 \quad \eta=1.0, T=10 \quad \eta \quad-\phi=0.8, T=20 \\
& \eta=0.8, T=10 \quad \eta=0.5, T=20 \quad \eta=1.0, T=20
\end{aligned}
$$

Figure S21: Results for $m=6$. Diversity as measured by the effective number of nodes $N_{\text {eff }}$ (the larger, the more egalitarian the indegree distribution): comparison between $R(k)+R P$-generated networks with different parameters $\eta=P / T=5 / 10,8 / 10,10 / 10,10 / 20,16 / 20,20 / 20$. The three columns correspond, from left to right, to $\beta=0.1,0.3,0.5$, respectively. The dots represent averages over 100 realizations; the error bars represent the standard error of the mean. 

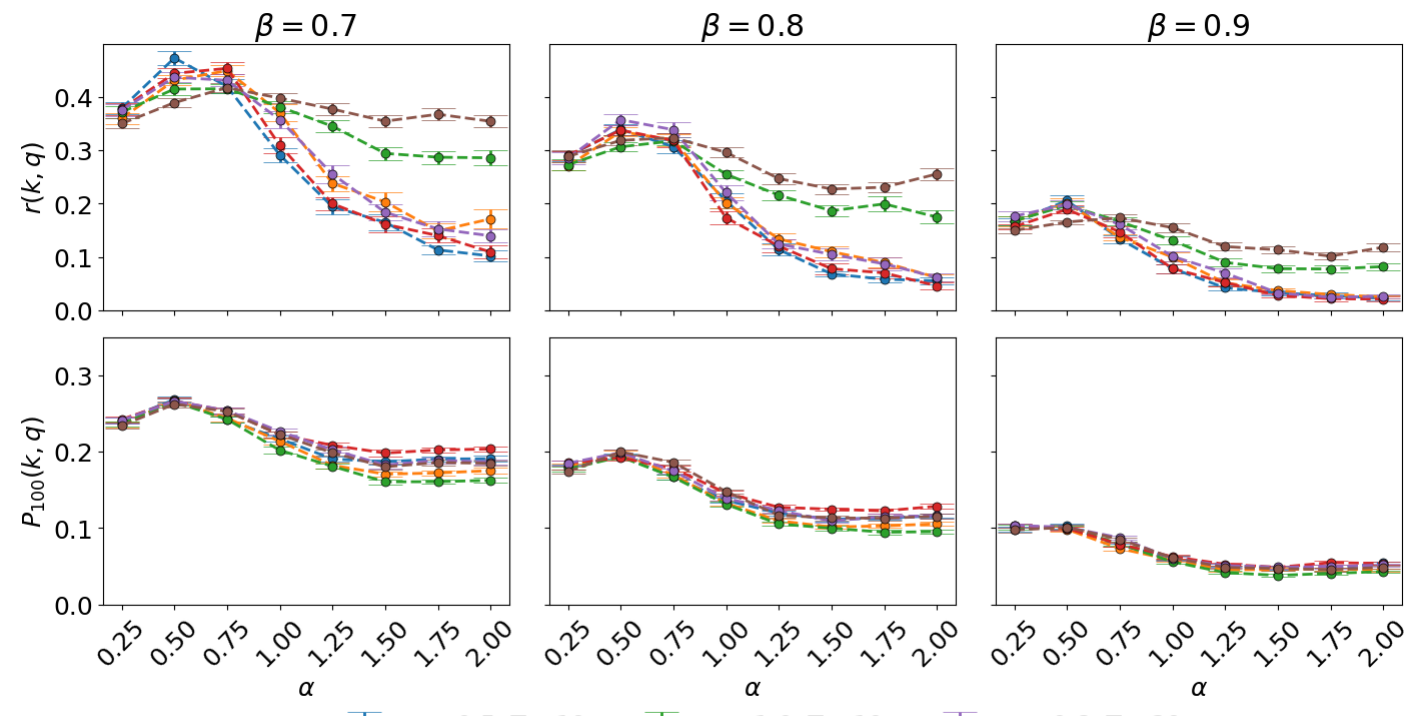

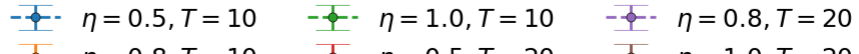

$\eta+\phi=0.8, T=10 \quad \eta=0.5, T=20 \quad-\phi=1.0, T=20$

Figure S22: Results for $m=6$. Quality promotion as measured by $r(k, q)$ (the Pearson's linear correlation between node indegree $k$ and node quality $q$-top panels), and $P_{100}(k, q)$ (the precision of node indegree $k$ in identifying the top-100 nodes by quality $q$-bottom panels): comparison between $R(k)+R P$-generated networks with different parameters $\eta=P / T=$ $5 / 10,8 / 10,10 / 10,10 / 20,16 / 20,20 / 20$. The three columns correspond, from left to right, to $\beta=0.7,0.8,0.9$, respectively. The dots represent averages over 100 realizations; the error bars represent the standard error of the mean. 

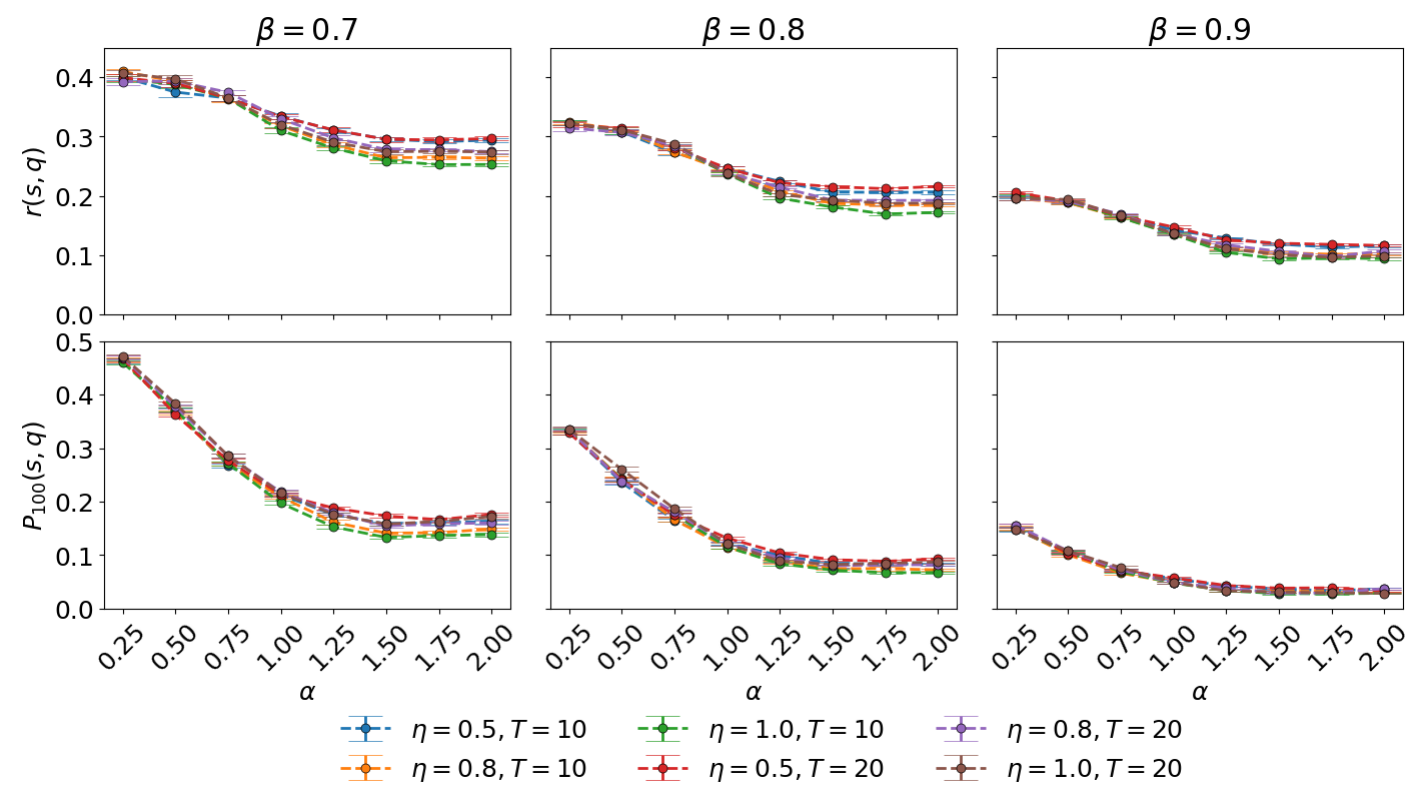

Figure S23: Results for $m=6$. Quality detection as measured by $r(s, q)$ (the Pearson's linear correlation between node score $s$ and node quality $q-$ top panels), and $P_{100}(s, q)$ (the precision of node score $s$ in identifying the top-100 nodes by quality $q$ - bottom panels): comparison between $R(k)+R P$-generated networks with different parameters $\eta=P / T=$ $5 / 10,8 / 10,10 / 10,10 / 20,16 / 20,20 / 20$. The three columns correspond, from left to right, to $\beta=0.7,0.8,0.9$, respectively. The dots represent averages over 100 realizations; the error bars represent the standard error of the mean. 

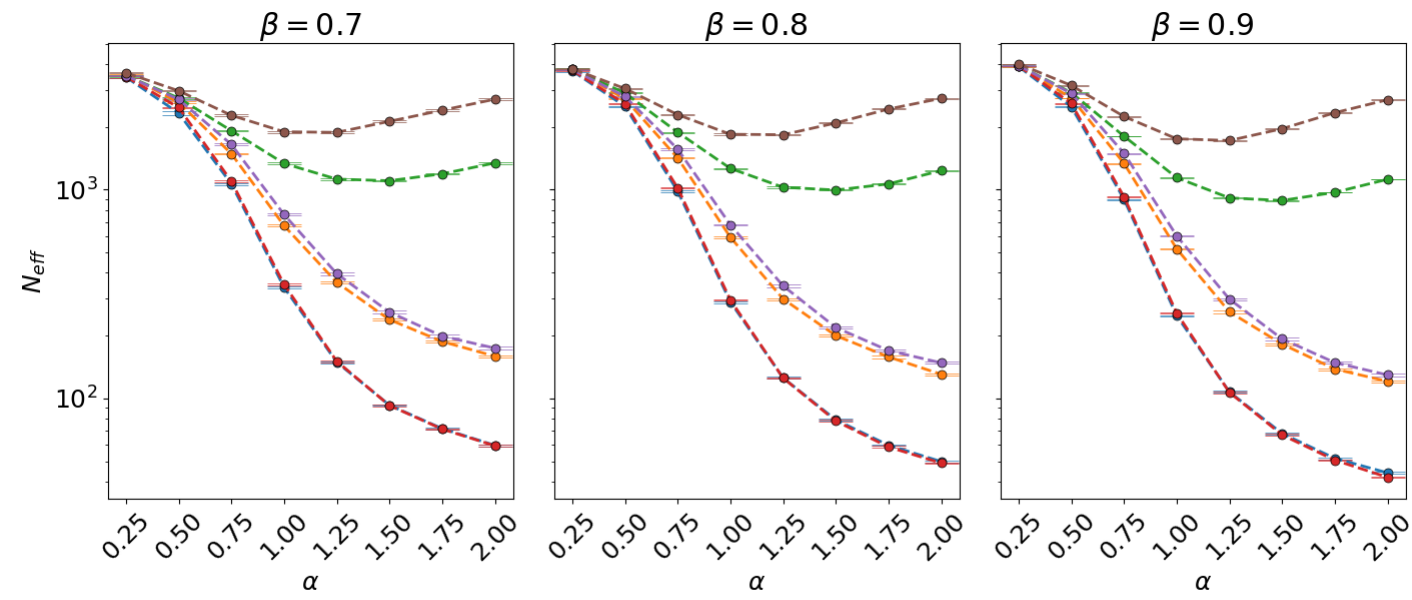

$$
\begin{aligned}
& \eta=0.5, T=10 \quad \eta=1.0, T=10 \quad \eta \quad-\phi=0.8, T=20 \\
& \eta=0.8, T=10 \quad \eta=0.5, T=20 \quad \eta=1.0, T=20
\end{aligned}
$$

Figure S24: Results for $m=6$. Diversity as measured by the effective number of nodes $N_{\text {eff }}$ (the larger, the more egalitarian the indegree distribution): comparison between $R(k)+R P$-generated networks with different parameters $\eta=P / T=5 / 10,8 / 10,10 / 10,10 / 20,16 / 20,20 / 20$. The three columns correspond, from left to right, to $\beta=0.7,0.8,0.9$, respectively. The dots represent averages over 100 realizations; the error bars represent the standard error of the mean. 
A)
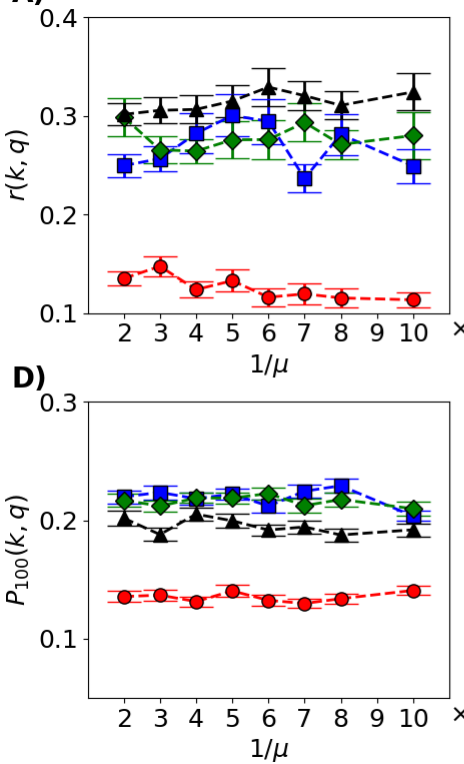

B)

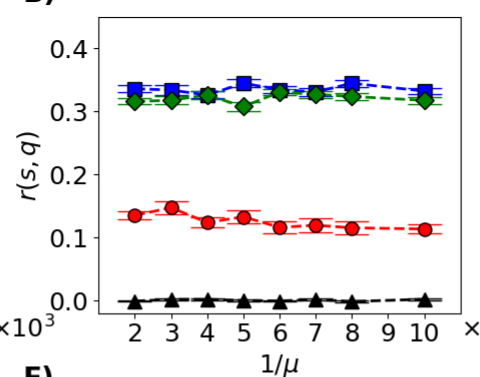

E)

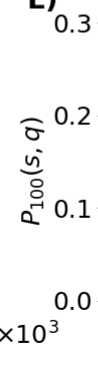

C)
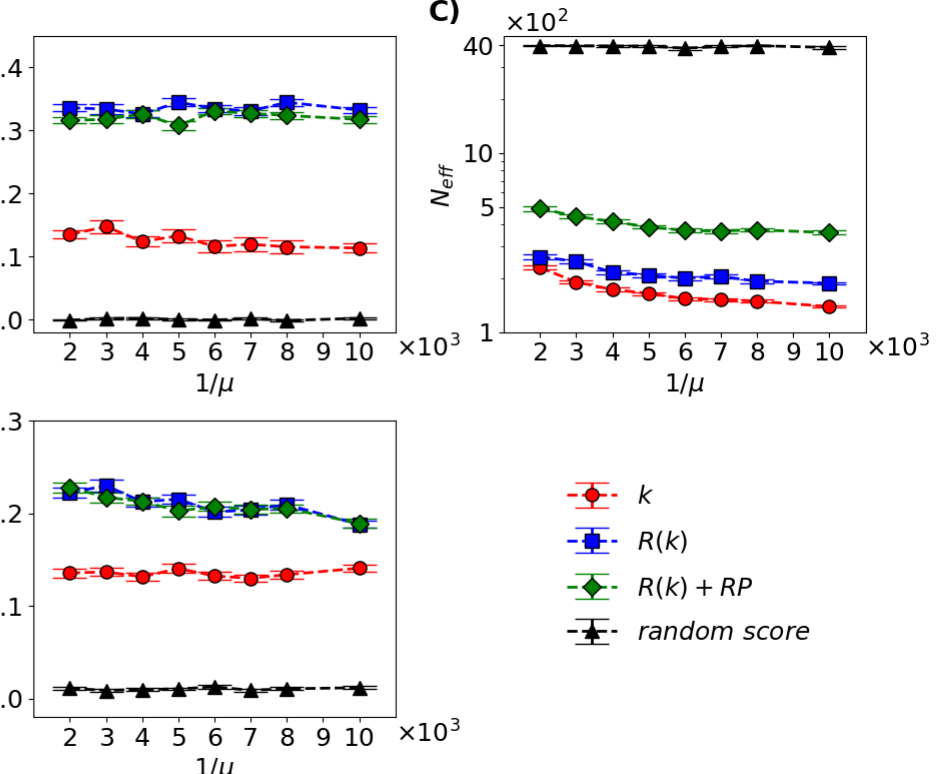

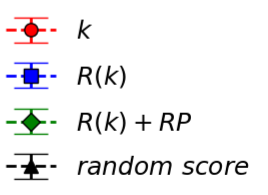

Figure S25: Results for model including node removal, $m=6$. The impact of node removal on quality promotion, quality detection, popularity diversity. For $\alpha=1.0$ and $\beta=0.7$, we show five network properties as a function of the inverse $A^{*}=\mu^{-1}$ of the removal probability: (A) the Pearson's correlation between node indegree and node quality; (B) the Pearson's correlation between node score and node quality; (C) the effective number of nodes, $N_{\text {eff }}$; (D) the indegree's precision in identifying the top-100 nodes by quality; (E) the score's precision in identifying the top-100 nodes by quality. Different lines correspond to the networks generated with different algorithms. The dots represent averages over 50 realizations; the error bars represent the standard error of the mean. 


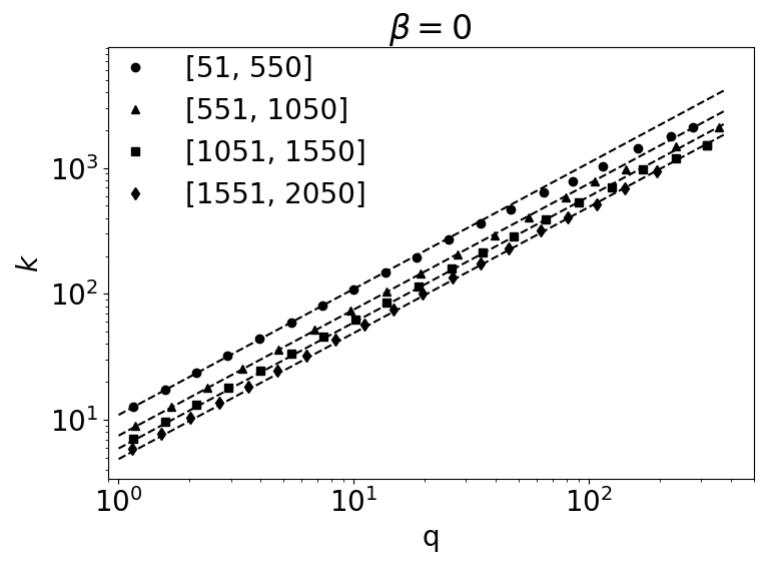

Figure S26: Results for $m=6$. The relation between node indegree and quality in networks of $N=10,000$ nodes generated with $\beta=0$. To factor out the dependence of node indegree on node age, we group together nodes of similar age - we represent here the results for four such age groups. The analytic lines well match the results of numerical simulations for sufficiently small $q$ values.
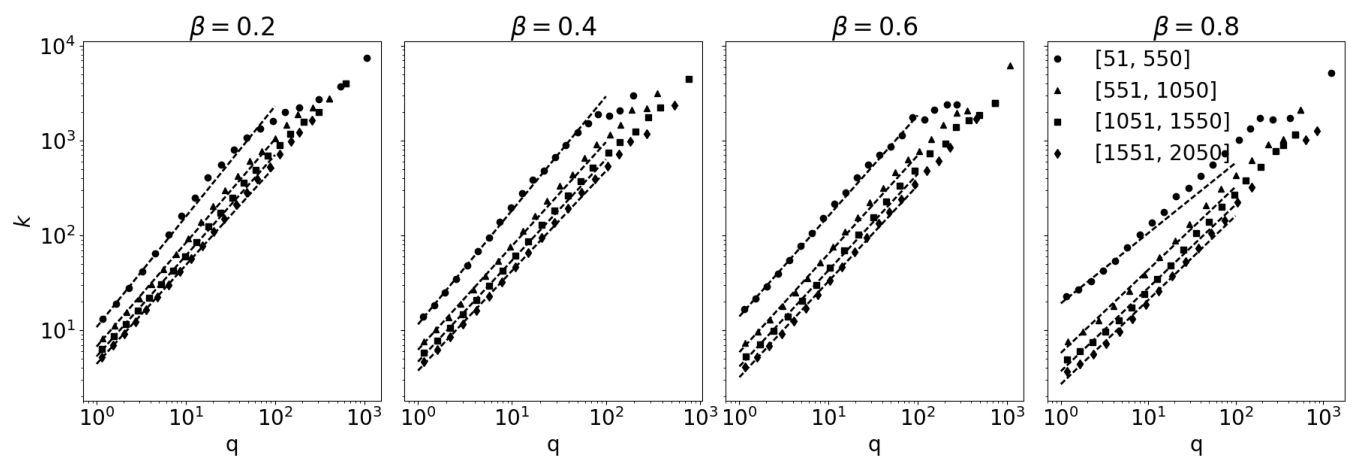

Figure S27: Results for $m=6$. The relation between node indegree and quality in networks of $N=10,000$ nodes generated with $\beta=0,2,0.4,0.6,0.8$. To factor out the dependence of node indegree on node age, we group together nodes of similar age - we represent here the results for four such age groups. The results of a power-law fit (see Table S1) well match the numerical results for small $q$ values. 


\begin{tabular}{|c|c|c|c|c|c|c|c|c|}
\hline \multirow{2}{*}{ Age group } & \multicolumn{2}{|c|}{$\beta=0.2$} & \multicolumn{2}{c|}{$\beta=0.4$} & \multicolumn{2}{c|}{$\beta=0.6$} & \multicolumn{2}{c|}{$\beta=0.8$} \\
\cline { 2 - 9 } & $\mathrm{A}$ & $\delta$ & $\mathrm{A}$ & $\delta$ & $\mathrm{A}$ & $\delta$ & $\mathrm{A}$ & $\delta$ \\
\hline$[51,550]$ & 10.80 & 1.16 & 11.54 & 1.20 & 14.02 & 1.06 & 19.23 & 0.74 \\
{$[551,1050]$} & 6.73 & 1.09 & 6.24 & 1.09 & 5.89 & 1.04 & 5.78 & 0.88 \\
{$[1051,1550]$} & 5.31 & 1.06 & 4.68 & 1.07 & 4.16 & 1.01 & 3.71 & 0.88 \\
{$[1551,2050]$} & 4.42 & 1.04 & 3.76 & 1.05 & 3.20 & 1.01 & 2.71 & 0.89 \\
\hline
\end{tabular}

Table S1: Results for $m=6$. Results of the fit of the relation between $k$ and $q$ (Fig. S17) using $\bar{k}=A_{i} q^{\delta_{i}}$; To account for temporal effects, we split the nodes into groups of 500 nodes based on their age (e.g., $[551,1050])$. For each age group, the fit is performed as a least-squares linear regression of the relation $\log k=\log A_{i}+\delta_{i} \log q$, for $q \leq 100$. We find that the fit results well match the numerical results (see Fig. S17), and the exponent $\delta_{i}$ depends weakly on the age group $i$. 